\title{
INPP4B promotes leukemia by restricting leukemic stem cell differentiation through regulation of lysosomal functions
}

\section{Authors:}

John F. Woolley ${ }^{1,2, \#^{*}}$, Keyue Chen ${ }^{1}$, Gizem E. Genc ${ }^{3}$, Daniel K.C. Lee ${ }^{1}$, Irakli Dzneladze ${ }^{2}$, Ruijuan $\mathrm{He}^{2}$, Martino M. Gabra ${ }^{1}$, Golam T. Saffi ${ }^{1}$, Meong Hi Son ${ }^{1,2,}$, Erwin M. Schoof ${ }^{2}$, Stephanie Z. Xie ${ }^{2}$, Emily M. Mangialardi ${ }^{1}$, Max Kotlyar ${ }^{4}$, Ayesha Rashid ${ }^{2}$, Miki. S. Gams ${ }^{5,6}$, Jean Vacher ${ }^{7}$, Cynthia J. Guidos ${ }^{5,6}$, Igor Jurisica $^{4,8,9,10}$, John E. Dick ${ }^{2,11,12}$, Roberto J. Botelho ${ }^{3}$, Mark D. Minden ${ }^{2,7}$, and Leonardo Salmena ${ }^{1,2,11^{*}}$

\section{Affiliations:}

${ }^{1}$ Department of Pharmacology \& Toxicology, University of Toronto, Toronto, Ontario M5S 1A8, Canada

${ }^{2}$ Princess Margaret Cancer Centre, University Health Network, Toronto, Ontario M5G 1L7, Canada

${ }^{3}$ Department of Chemistry and Biology, Ryerson University, Toronto, Ontario M5B 2K3, Canada

${ }^{4}$ Osteoarthritis Research Program, Division of Orthopedic Surgery, Schroeder Arthritis Institute, University Health Network; Data Science Discovery Centre for Chronic Diseases, Krembil Research Institute, University Health Network, Toronto, Ontario M5T 0S8, Canada.

${ }^{5}$ Developmental \& Stem Cell Biology Program, Hospital for Sick Children Research Institute, Toronto, Ontario, Canada.

${ }^{6}$ Department of Immunology, University of Toronto, Toronto, Ontario, Canada.

${ }^{7}$ Institut de Recherches Cliniques de Montréal (IRCM), Département de Médecine, Université de Montréal, Montréal, Québec H2W 1R7, Canada

${ }^{8}$ Department of Computer Science, University of Toronto, Toronto, Ontario M5T 3A1, Canada

${ }^{9}$ Departments of Medical Biophysics, University of Toronto, Toronto, Ontario M5G 1L7, Canada

${ }^{10}$ Institute of Neuroimmunology, Slovak Academy of Sciences, Bratislava, Slovakia

${ }^{11}$ Department of Molecular Genetics, University of Toronto, Toronto, Ontario M5S 1A8, Canada

${ }^{12}$ Ontario Institute for Cancer Research, Toronto, Ontario M5G 0A3, Canada

${ }^{11}$ Lead Contact

\section{Author list footnotes:}

${ }^{\ddagger}$ Present address: Department of Pharmacology \& Therapeutics, Institute of Systems, Molecular and Integrative Biology, University of Liverpool, UK.

${ }^{\S}$ Present address: Department of Pediatrics, Samsung Medical Center, Sungkyunkwan University School of Medicine, Seoul, Korea

*Correspondence: john.woolley@ liverpool.ac.uk (J.F.W); leonardo.salmena@utoronto.ca (L.S.) 


\section{Running Title: INPP4B controls leukemia stem cells by lysosome regulation}

\section{Summary (150 max)}

Despite an increased understanding of leukemogenesis, specific mechanisms that underlie 'stemness' in leukemia remain largely undefined. Here, we report a novel pathway which regulates leukemic differentiation through control of lysosomal biology. We show that disruption of INPP4B

results in dysregulated lysosomal gene networks, reduced lysosomal numbers and proteolytic capacity in leukemia. Inpp4b-deficient HSCs and LSCs are functionally compromised. Inpp4b-deficient leukemia models develop more differentiated leukemias with reduced disease initiating potential, and improved overall survival compared to Inpp $4 b$-expressing leukemias. Together, our data is consistent with a model where INPP4B restricts differentiation of LSCs through regulation of lysosomal function. These data provide a mechanism to explain the association of INPP4B with aggressive AML and highlight avenues for LSC-specific leukemia therapies.

\section{Keywords}

INPP4B, AML, LSC, lysosome, differentiation

\section{Statement of Significance ( 50 words)}

This work is the first to show that the phosphoinositide phosphatase INPP4B regulates stemness and differentiation in AML, through a previously unknown role in lysosome function. Herein we describe a mechanism to explain why INPP4B overexpression is associated with poor clinical outcome in AML. 


\section{Introduction}

Acute myeloid leukemia (AML) is an aggressive malignancy of the bone marrow characterized by dysregulated proliferation of immature myeloid cells and dismal survival rates (Estey and Döhner, 2006; Klepin et al., 2014; Löwenberg et al., 1999). Most AML patients who receive intensive chemotherapy achieve a significant clinical response, however a majority will relapse within 5 years and succumb to their disease (Grimwade et al., 2010; Walter et al., 2015). Thus, AML remains a largely incurable cancer, due to disease relapse and chemoresistance.

AML has been shown to exist as a cellular hierarchy, with leukemic stem cells (LSC) at the pinnacle which possess 'stemness' attributes including a capacity for self-renewal, differentiation, longterm clonal propagation and quiescence, which all serve to continuously sustain a bulk of malignant undifferentiated myeloid 'blast' cells that define AML (Dick, 2008). It has been demonstrated that AML patients with greater LSC content and more stem-like disease have worse clinical outcomes (Eppert et al., 2011; Ng et al., 2016; van Rhenen et al., 2005; Shlush et al., 2017). Thus, in the absence of LSCeradicating therapies it is not surprising that relapse occurs in the majority of AML patients. Despite an increased understanding of AML and leukemogenesis over the past decades, specific molecular machinery that underlie 'stemness' in AML remains largely undefined.

The lysosome is a major platform for many cellular signaling pathways including cell growth, division, differentiation (Inpanathan and Botelho, 2019; Lawrence and Zoncu, 2019); however, distinct lysosomal activities in stem cells are still emerging. Functional lysosomes in neuronal stem cells are needed to mitigate protein-aggregate accumulation, a consequence which would otherwise increase aging, reduce stem functions and lead to differentiation of these cells (Leeman et al., 2018). Emerging studies in hematopoietic stem cells (HSC) suggest that lysosomes are asymmetrically inherited and thus predictive of future daughter cell fates (Loeffler et al., 2019); pharmacological inhibition of the lysosomal v-ATPase can positively impacted mouse HSC engraftment (Liang et al., 2020), lysosomes have been shown to coordinate the cell cycle and metabolic machinery of LT-HSC through their ability 
to sense and respond to diverse signaling cues (García-Prat et al., 2021). In leukemia, increased lysosomal mass and biogenesis in leukemic progenitor cells selectively sensitizes AML cells to lysosomal inhibitors or disruptors (Sukhai et al. 2013; Bernard et al. 2015). Nevertheless, roles for lysosome in LSC and leukemic differentiation remain largely unknown.

We previously reported that Inositol Polyphosphate-4-Phosphatase Type II (INPP4B), a lipid phosphatase that hydrolyzes phosphatidylinositol-3,4-bisphosphate $\left[\operatorname{Ptd} \operatorname{Ins}(3,4) \mathrm{P}_{2}\right]$ to generate phosphatidylinositol-3-monophosphate [PtdIns(3)P] demonstrates elevated expression levels in the leukemic blasts of approximately one quarter of all AML patients (Dzneladze et al., 2015). Studies from our group and others in AML (Dzneladze et al., 2015; Jin et al., 2018; Recher, 2015; Rijal et al., 2015; Wang et al., 2016; Zhang et al., 2017, 2019) and other malignancies (Chi et al., 2015; Gasser et al., 2014; Guo et al., 2016) demonstrate that INPP4B possesses tumour promoting and oncogenic functions. These findings prompted us to investigate specific roles of INPP4B in hematopoiesis and leukemogenesis. Herein we report novel functions for INPP4B in hematopoietic stemness and a role for INPP4B in restricting the differentiation of LSC through the regulation of lysosome biology and function. 


\section{Results}

\section{Hematopoietic stem and progenitor cell populations express elevated levels of INPP4B}

To investigate a role for INPP4B in hematopoiesis, we first interrogated expression datasets derived from purified human (Jung et al., 2015; Laurenti et al., 2013, 2015; Lechman et al., 2016; Notta et al., 2016; Novershtern et al., 2011; Rapin et al., 2014) and mouse (Lara-Astiaso et al., 2014; Pietras et al., 2015) hematopoietic stem and progenitor cell (HSPC) populations datasets. We observed that the expression of INPP4B is consistently highest in HSPC populations with the greatest pluripotency and self-renewal potential [eg. long-term (LT)-HSCs, short-term (ST)-HSCs and multipotent progenitor cells (MPPs)]; progressively lower levels of expression were observed in committed progenitors and terminally differentiated myeloid lineage cells including granulocytes and monocytes (Fig. 1A and Supplementary 1A). Similarly, in murine datasets, Inpp $4 b$ expression was highest in LT-HSCs and bulk HSCs, with lower levels found in lineage restricted HSPCs and terminally differentiated populations (Fig. 1B and Supplementary 1B). These data indicate that INPP4B expression is associated with HSPC pluripotency, pointing to a putative role for INPP4B in HSC maintenance (Fig. 1C).

\section{Inpp4b deficiency leads to alterations in hematopoietic progenitor populations}

To study Inpp $4 b$ function in HSPCs, we employed a constitutive Inpp $4 b$-knockout mouse model $\left(\operatorname{Inpp} 4 b^{-/}\right)$generated by Vacher and colleagues (Ferron et al., 2011). Inpp $4 b^{-/}$and Inpp $4 b^{+/-}$mice are viable and born at the expected Mendelian frequencies. We did not observe the occurrence of any cancers or premature lethality in $\operatorname{Inpp} 4 b^{-/-}$or $\operatorname{Inpp} 4 b^{+/-}$mice up to and beyond 2 years of age consistent with other published Inpp4b mouse models (Kofuji et al., 2015; Li Chew et al., 2015). In this study we focused our analysis of the knockout animals to the hematological system. Gross hematological phenotypes in 6-10 weeks old Inpp $4 b^{-/-}$or Inpp $4 b^{+/+}$mice demonstrated no measurable differences in 
total bone marrow cellularity, circulating white blood cell counts, lymphocytes, monocytes, neutrophils, red blood cells and platelet numbers in peripheral blood (Fig. Supplementary 1C, 1D).

In contrast, flow cytometric analysis of progenitor populations from adult $\operatorname{Inpp} 4 b^{+/+}$and $\operatorname{Inpp} 4 b^{-}$ ${ }^{\prime}$ mice (>6 weeks of age) revealed a marked reduction in the number of LSK $\left(\mathrm{Lin}{ }^{-} \mathrm{Sca}-1^{+} \mathrm{c}-\mathrm{Kit}^{+}\right.$), shortterm HSCs (ST-HSC; CD34 ${ }^{+} \mathrm{CD} 150^{+} \mathrm{CD} 48^{-} \mathrm{LSK}$ ) and long-term HSC (LT-HSC; CD34-CD150 CD48LSK or EPCR ${ }^{+}$LSK) (Fig. 1D,E; Fig. Supplementary 1E,F), and a suggestive reduction in multipotent progenitors (MPP) (Fig. 1E) in $\operatorname{Inpp} 4 b^{-/-}$as compared to Inpp $4 b^{+/+}$mice. Granulocyte monocyte progenitors (GMP) were significantly reduced, and megakaryocyte erythrocyte progenitors (MEP) were significantly increased in Inpp $4 b^{-/-}$bone marrow compared to Inpp $4 b^{+/+}$bone marrow. No differences were observed in common myeloid progenitor (CMP) and lymphomyeloid-primed progenitor (LMPP) fractions (Fig 1F). Together, these findings suggest a role for Inpp $4 b$ expression in the homeostasis of HSPC populations.

\section{Hematopoietic stem cells have reduced function in Inpp $4 b$ knock-out mice}

To measure functional consequences of Inpp4b-deficiency on defining hallmarks of HSC including self-renewal, differentiation, and long-term clonal propagation we tested wild type and Inpp $4 b$-deficient HSC functionality both in vitro and in vivo. First, we evaluated the in vitro colony forming and self-renewal potential of bone marrow cells from Inpp $4 b^{+/+}$and $\operatorname{Inpp} 4 b^{-/}$mice using colony-forming cell (CFC) assays with serial replating every seven days for a total of 4 weeks (Fig. 1G). Seven days after the initial plating, we observed no difference in the number of total colonies between Inpp $4 b^{+/+}$and Inpp $4 b^{-/-}$colony numbers. However, upon subsequent re-plating of colonies, total colony numbers from Inpp $4 b^{-/}$bone marrow were significantly reduced (day 14: $126 \pm 8.4$ vs $94 \pm 8.2$ ), with further reduction evident after the third plating (day 21: $105 \pm 15.0$ vs $14 \pm 3.9$ ) indicating a premature collapse of colony forming capacity of Inpp $4 b^{-/-}$bone marrow cells. The reduction in total colony 
numbers at day 14 between $\operatorname{Inpp} 4 b^{+/+}$and $\operatorname{Inpp} 4 b^{-/-}$was predominantly a result of fewer CFU-M colonies in Inpp $4 b^{-/-}$compared to other lineages (Fig. Supplementary 1G) suggesting that Inpp4bdeficiency leads to a defect in the differentiation of myeloid-committed precursors, particularly of the monocyte lineage. By day 21, there was premature collapse of all lineages in Inpp $4 b^{-/-}$progenitors (Fig. 1G, Fig. Supplementary 1G).

To investigate whether Inpp4b-deficiency impacts the repopulation potential of HSCs in vivo, we challenged Inpp $4 b^{+/+}$and Inpp $4 b^{-/}$mice with a myeloablation regimen of weekly i.p. injections of 5'fluorouracil (5'-FU). Survival analysis demonstrated that $\operatorname{Inpp} 4 b^{-/}$mice had a significantly reduced overall survival (median survival 24 days) compared to wild-type littermates $(P=0.008$; median survival 28 days) upon serial myeloablation (Fig. 1H). To estimate the relative quiescence of HSCs upon 5'-FU treatment in vivo, we performed Ki67 staining of sorted HSPCs $\left(\mathrm{Lin}^{-} \mathrm{Sca}-1^{+} \mathrm{Kit}^{+}\right) 48$ hours after a single acute treatment of $5^{\prime}$-FU. We observed that Inpp $4 b^{-/-}$HSPCs had a significant increase in cycling cells as compared to Inpp $4 b^{+/+}$HSPCs (Fig. 1I). Together, these data suggest that the induction of replicative-stress by serial myeloablation in $\operatorname{Inpp}_{4 b^{-/}}$mice leads to an aberrant escape-fromquiescence of hematopoietic progenitor associated with premature lethality.

Next, to evaluate the contribution of Inpp $4 b$ to the long-term repopulation capacity of HSPCs we carried out a serial competitive transplant assays with Inpp $4 b^{+/+}, \operatorname{Inpp} 4 b^{+/}$and Inpp $4 b^{-/}$bone marrow where LT-HSC output was determined as the relative contribution of CD45.1 (competitor HSPCs) and CD45.2 (test HSPCs) to the myeloid lineage over 20 weeks post-transplant, in 3 consecutive in vivo repopulation experiments (Fig. 1J, K, Fig. Supplementary 1H). Notably, at the end of the primary competitive transplant at week 20 we observed that unlike Inpp $4 b^{+/+}$HSPCs which contribute half $(50 \%)$ of total myeloid cells, Inpp $4 b^{-/-}(78.5 \%)$ and $\operatorname{Inpp} 4 b^{+/-}(70.0 \%)$ HSPCs outcompeted Inpp $4 b^{+/+}$ HSPCs. However, at the end of the experiment at 64 weeks, we observed that both $\operatorname{Inpp} 4 b^{+/}$and $\operatorname{Inpp}_{4 b^{-}}$

/ bone marrow cells demonstrated a near-complete loss of contribution to the myeloid compartment 
compared to Inpp $4 b^{+/+}$bone marrow, demonstrating that long-term hematopoietic reconstitution is significantly compromised with Inpp $4 b$-deficiency. The increased contribution of the $\operatorname{Inpp} 4 b^{-/-}$and Inpp $4 b^{+/}$cells at the first time point may be due to the increased rate of proliferation and failure of these cells to return to rest as observed in Fig 1I. At the end of the second transplant (44 weeks), Inpp $4 b^{+/+}$ HSPCs continue to contribute approximately half (44\%) of the total donor derived myeloid cells. However, in contrast to the earlier time point Inpp $4 b^{-/}$HSPCs demonstrate a reduced reconstitution capacity at $27 \%$ and $\operatorname{Inpp} 4 b^{+/-}$HSPCs continue to outcompete Inpp $4 b^{+/+}$at $73 \%$ of total myeloid cells. Taken together these data indicate that Inpp4b-deficient HSCs have a reduced capacity for long-term hematopoietic repopulation as a result of elevated sensitivity to replicative stress.

\section{INPP4B expression is associated with leukemic stemness gene networks}

Given the role we have demonstrated for INPP4B in hematopoiesis combined with our previous data demonstrating a role for INPP4B in AML prognosis (Dzneladze et al., 2015; Rijal et al., 2015) we reasoned that INPP4B may also play a role in leukemogenesis and LSC biology. Interestingly, INPP4B is one of forty-four genes enriched in HSCs and LSCs gene profiles(Eppert et al., 2011). Furthermore, we interrogated INPP4B expression in gene expression profiles from a total of 227 AML patient-derived cells sorted based on the expression of combinations of CD34 and CD38 surface markers ( $\mathrm{Ng}$ et al., 2016). We observed the highest levels of INPP4B transcript in $\mathrm{CD}^{+} 4^{+} \mathrm{CD}^{-} 8^{-}>\mathrm{CD} 34^{+} \mathrm{CD} 38^{+}>\mathrm{CD}^{-}$ $\mathrm{CD}^{-} 8^{-} \mathrm{CD}^{-} 4^{-} \mathrm{CD} 38^{+}$(Fig. 2A), where $\mathrm{CD}^{-} 4^{+} \mathrm{CD} 38^{-}$populations are considered to be enriched for LSC content compared to other AML subsets. In keeping with the notion that LSC and their capacity for disease reconstitution is not defined solely on the basis of the $\mathrm{CD} 34^{+} \mathrm{CD} 38^{-}$phenotype, we also interrogated datasets from LSC populations defined functionally through bone marrow transplant experiments (Eppert et al., 2011; Ng et al., 2016). We observed that gene expression profiles from 278 CD34/CD38 sorted populations derived from 78 AML patients (Ng et al., 2016) had significantly 
elevated levels of $I N P P 4 B$ expression and the $I N P P 4 B^{\text {high }}$ samples in functionally defined LSC had an odds ratio of $\sim 6$ for association with functionally defined $\mathrm{LSC}^{+}$populations (Chi-square $P$-value $<$ 0.0001) (Fig. 2B). Furthermore, using gene-set enrichment analysis (GSEA) we investigated the association of high INPP4B expression to the LSC17 signature genes ( $\mathrm{Ng}$ et al., 2016) in six public AML patient databases (Balgobind et al., 2011; Cancer Genome Atlas Research Network et al., 2013; Klein et al., 2009; Metzeler et al., 2008; Verhaak et al., 2009). We observed that the LSC-17 signature was significantly enriched in patient samples with high INPP4B expression in all AML patient databases tested (Fig. 2C \& Supplementary Fig. 2). Altogether, our analyses of INPP4B gene expression in AML subsets and functionally defined LSC populations are the first data pointing to an association between INPP4B and leukemic stemness.

\section{Inpp4b-deficiency leads to increased disease latency and a more differentiated leukemia}

To investigate a direct role for Inpp $4 b$ in leukemogenesis and LSC function in vivo, we generated a model of Inpp $4 b$-deficient leukemia by transducing Inpp $4 b^{-/-}$LSK cells and control Inpp $4 b^{+/+}$LSK $^{-}$ cells with MSCV-MLL-AF9-Venus retrovirus. Venus ${ }^{+} \operatorname{Inpp} 4 b^{-/-}$and Venus ${ }^{+}$Inpp $_{4} b^{+/+}$LSK cells were transplanted by tail vein injection into sub-lethally irradiated syngeneic host C57BL/6 mice to promote the development of primary leukemias (Supplementary Fig. 3A). Notably, all host animals transplanted with Inpp $4 b^{+/+}$MLL-AF9-infected cells succumbed to leukemia associated disease, however $\sim 40 \%$ of the host animals transplanted with Inpp $4 b^{-/}-M L L-A F 9$ leukemia cells survived beyond 300 days suggesting that Inpp $4 b^{-/-}$-leukemias have decreased potential to kill the animals either due to overwhelming leukemia or failure of normal hematopoiesis (Fig. 3A). In support of this, secondary transplants of $M L L-A F 9$ leukemias (Fig. 3B) also demonstrated a significant increase in disease latency for the $\operatorname{Inpp} 4 b^{-/-}-M L L-A F 9$ when compared to the Inpp $4 b^{+/+}-M L L-A F 9$ (median survival of 76 vs. 41 days respectively; $\mathrm{p}=0.0246$ ). Furthermore, limiting dilution cell transplantation assays (LDA) revealed 
that $\operatorname{Inpp} 4 b^{-/-}-M L L-A F 9$ leukemias have a significant decrease $(1 / 147 v s 1 / 300 P=0.0331)$ in leukemic initiating cell (LIC) frequency (Fig. 3C).

We used mass cytometry to evaluate expression of 15 hematopoietic differentiation markers. In contrast to WT bone marrow, MLL-AF9 leukemias had very few eyrthroid and T/B lymphocytes (data not shown). Instead, they consisted predominantly of $\mathrm{CD} 11 \mathrm{~b}^{+} \mathrm{CD} 16 / 32^{+} \mathrm{Gr}^{-}$cells that lacked Sca-1 and CD150, indicative of myelomonocytic differentiation (Fig. 3D, E and data not shown). However, a significantly higher percentage of Inpp $4 b^{-/}$leukemic cells also expressed Gr1, suggesting that Inpp $4 b$ deficiency allowed some granulocytic differentiation. The Inpp $4 b^{-/-}$leukemia cells had relatively more CD16/32 $2^{+} \mathrm{CD} 117^{+}$cells (Fig. 3E), but this difference was not significant. In contrast, Inpp $4 b^{-/}$and Inpp $4 b^{+/+}-M L L-A F 9$ expressed significantly different levels of CD24, CD44 and CD16/32 (Fig. 3F, G). These data indicate that Inpp $4 b$ deficiency alters the differentiation status of $M L L-A F 9$-induced myeloid leukemia.

To further support these observations, Leukemia forming cell (LFC) assays carried out with primary Inpp $4 b^{+/+}$and Inpp $4 b^{-/-} M L L-A F 9$ leukemic cells demonstrated that Inpp $4 b$-deficiency leads to a significant decrease in total leukemic colonies formed (Fig. 3H). Furthermore, Inpp4b-deficiency leads to a decrease in the percentage of type-I colonies (spherical with defined border, resembling primitive hematopoietic colony formation) countered by an increase in more differentiated type-II colonies (diffuse, lacking a defined border) (Fig. 3H) (Krivtsov et al., 2006; Somervaille and Cleary, 2006). Finally, Wright-Giemsa staining of leukemic smears revealed a more differentiated blast cell phenotype in $\operatorname{Inpp}_{4 b^{-/}} M L L-A F 9$ as determined by an elevated proportion of multi-lobed, horseshoe-shaped or diffuse nuclei (Fig. 3I). Only $16.9 \%$ of Inpp $4 b^{+/+} M L L-A F 9$ leukemia cells had a differentiated morphology, whereas $51.6 \%$ of $\operatorname{Inpp} 4 b^{-/-}-M L L-A F 9$ leukemias had a differentiated phenotype. Collectively, these data indicate that Inpp4b-deficiency in a $M L L-A F 9$ leukemia model generates leukemias with decreased colony forming potential and altered morphology, which is consistent with the 
observed decrease in leukemogenic potential and altered differentiation status (Somervaille and Cleary, 2006).

\section{Identification of transcriptional networks perturbed by Inpp $4 b$ loss in leukemia cells}

To identify the consequences of Inpp $4 b$ deficiency in leukemic transcriptional networks we performed RNA sequencing (RNA-seq) on freshly isolated bone marrow from Inpp $4 b^{+/+}$and $\operatorname{Inpp} 4 b^{-/-}$ MLL-AF9 leukemias. RNA-seq revealed a total of 5462 differentially expressed genes between Inpp $4 b^{+/+}$and Inpp $4 b^{-/-}$MLL-AF9 leukemias (2434 downregulated, 3028 upregulated; FDR < 0.05; Supplementary Fig. 4A,B, Supplementary Table 1).

To identify biological processes most significantly influenced by Inpp $4 b$ deficiency, we performed gene ontology (GO) and Gene Set Enrichment Analysis (GSEA) on the differentially expressed gene list (Fig. 4A,B; Supplementary Table 2). Both analyses revealed enrichment of lysosomal gene sets (eg. 64 out of 124 genes in the Kyoto Encyclopedia of Genes and Genomes $\left(\right.$ KEGG) lysosomal gene signature; corrected $P$-value $\left.=3.62 \times 10^{-06}\right)$. Gene profiling demonstrated that a disproportionately large number of KEGG lysosomal gene transcripts were downregulated in Inpp $4 b^{-/}$ $M L L-A F 9$ gene profiles compared to Inpp $4 b^{+/+}$controls (Fig. 4C). Using data generated by STRING-db, we observed that the KEGG lysosome signature gene set could be subdivided into three protein interaction networks: trans-golgi network trafficking proteins, vacuolar ATPases and lysosome-active proteins and transporters. Although we observed loss of gene expression in all three subdivisions, the most notable losses were strongly associated with lysosome-active proteins and transporters including several cathepsins (Ctss, Ctsc and Ctsf), lysosomal transporters (Slc11al) and other proteases (Lgmn, Psap, Lipa) (Fig. 4C,D). To further the association between Inpp4b expression with lysosomal transcripts (Fig. 4E), we performed GSEA using an independent set of transcription factor EB (TFEB)regulated lysosomal genes from the Coordinated Lysosomal Expression and Regulation (CLEAR) 
network (Fig. 4F) with similar results (Sardiello et al., 2009). Together these results point to a role for Inpp4b in regulating lysosomal biology through regulation of lysosomal gene expression.

To further support the notion that Inpp $4 b$ deficiency was associated with a phenotypically differentiated leukemia we investigated the presence of changes in genes and networks associated with leukemic stemness or myeloid differentiation. Using GSEA we observed that there were significant changes in the LSC17 gene signature (Fig 4G), a myeloid cell development signature and an osteoclast differentiation signature (Supplementary Fig 4C,D) in Inpp4b-deficiency (Mootha et al., 2003; Ng et al., 2016; Subramanian et al., 2005). These findings indicate that Inpp4b-deficiency in $M L L-A F 9$ leukemia significantly alters gene transcription networks associated with lysosome biology, myeloid stemness and myeloid lineage differentiation.

\section{INPP4B expression in human AML is associated with a lysosomal gene signature}

Given our results linking Inpp4b-deficiency with disrupted lysosomal gene networks in murine leukemia, we investigated the presence of an orthologous association in human leukemia. For this we interrogated the TCGA-LAML and Verhaak-AML patient datasets, two of the largest publicly available AML datasets using GSEA (Cancer Genome Atlas Research Network et al., 2013; Mootha et al., 2003; Subramanian et al., 2005; Verhaak et al., 2009). Using a previously established optimal cutoff of $75 \%$ for INPP4B high/low expression and patient outcome in AML datasets as determined by our subgroup identifier (SubID) tool (Dzneladze et al., 2015, 2018), we identified the top 20 most enriched KEGGdefined gene sets associated with INPP4B expression. Notably, the lysosome, and other lysosomerelated gene sets including glycosaminoglycan degradation and other glycan degradation gene sets were ranked among the top significantly enriched gene sets in both TCGA (NES=1.74, NOM $p=0.02)$ and Verhaak (NES=1.90, NOM $p=0.02$ ) datasets (Fig 5A-D, Supplemental Fig 5A,B). Similar associations with the KEGG lysosomal gene set were observed in additional AML patient datasets from 
studies by Valk (NES=1.92, NOM $p<0.0001)$ and Wouters $(\mathrm{NES}=1.56$, NOM $p=0.02)($ Fig 5E,F;

Supplemental Fig 5C,D)(Valk et al., 2004; Wouters et al., 2009). Furthermore, among the 121 genes which comprise the lysosome gene set, 78 genes were classified as leading edge genes (LEGs) in at least one of the TCGA, Verhaak, Valk and Wouters datasets, and a core set of 30 LEGs were present in all 4 datasets (Fig 5G,H). Together, the results of our analysis of public AML datasets support our results in murine leukemia models and show that the expression of INPP4B and core lysosomal genes are associated across various AML patient datasets.

\section{INPP4B regulates lysosomal biology}

To substantiate our transcriptional and correlative data, we investigated a direct role for INPP4B in lysosome biology by measuring the consequences of INPP4B overexpression or knockdown on lysosomal characteristics in cancer cell lines. For these studies we generated inducible INPP4BU2OS osteosarcoma cells by inserting a donor vector sequence containing an inducible TRE3G-INPP4B and constitutive Tet-On 3G expression cassettes into the AAVS1 safe harbor locus by CRISPR genome editing (Mandegar et al., 2016). Lysosomal Associated Membrane Protein 1 (LAMP1), a type I membrane protein possessing a short cytosolic C-terminus harboring an endo-lysosomal targeting sequence, was used as a representative lysosomal marker (Bagshaw et al., 2005a, 2005b; Callahan et al., 2009). We evaluated cytoplasmic LAMP-1 puncta in the presence and absence of INPP4B induction using $100 \mathrm{ng} / \mathrm{mL}$ of doxycycline (Fig 6A). Upon induction of INPP4B, we observed a significant increase in the number of LAMP1 puncta (Fig 6A) and an increase in the total cellular intensity of LAMP1 staining (Supplemental Fig 6A). Conversely, siRNA-knockdown of INPP4B (Fig 6B) was associated with significantly decreased number of LAMP1 puncta (Fig 6B) and decreased intensity (Supplemental Fig 6B) compared to non-targeting siRNA (siNC; Fig 6B). 
The cellular positioning of lysosomes also plays an important role in lysosomal function (Cabukusta and Neefjes, 2018; Korolchuk et al., 2011; Pu et al., 2016; Settembre et al., 2012). Thus, we measured the extent of lysosomal dispersion in INPP4B overexpressing and knockdown cells by demarcating the cytoplasm of cells into 4 shells beginning with a perinuclear shell (Fig 6C). We observed that INPP4B overexpression led to an increased number of LAMP1 puncta in all peripheral shells, with no change in the inner perinuclear shell (Fig 6D). Conversely, LAMP1 dispersion upon INPP4B knockdown with INPP4B-siRNA did not significantly change lysosomal distribution compared to control cells (Fig 6E). To measure a role for INPP4B in regulating the proteolytic capacity of lysosomes, we incubated cells with (DQ)-BSA-Green, a quenched dye which generates bright fluorescence upon proteolysis within lysosomes. In these experiments, cells were allowed to simultaneously endocytose DQ-BSA-Green and fluorescent-tagged Dextran-Red. The latter is always fluorescent and can be normalized against DQ-BSA to control for any differences in the endocytosis rate of DQ-BSA, which in turn would affect DQ-BSA fluorescence. We observed that INPP4B overexpression resulted in activation of significantly greater levels of DQ-BSA fluorescence compared to controls (Fig 6F). Conversely, decreased DQ-BSA fluorescence was measured in INPP4Bknockdown cells when compared to controls (Fig 6G). Together, these results are the first demonstration of a role for INPP4B in regulating lysosomal content, dispersion and proteolytic function.

To provide evidence for a direct role for INPP4B on lysosomes, we examined whether INPP4B can localize to lysosomes. For these experiments, first we overexpressed mCherry-INPP4B in U2OS cells and measured colocalization to lysosomes labelled with Lucifer Yellow. We observed a significant increase in INPP4B-mCherry localization to lysosomes when compared to empty-mCherry vector (Supplemental Fig 6C-D). Colocalization of INPP4B-mCherry with Lucifer Yellow stained lysosomes was measured to be stable for at least 20 min (Supplemental Fig 6E,F). Furthermore, immunoblotting of lysosomal immunoprecipitations confirmed the interaction of overexpressed INPP4B with lysosomes (Supplemental Fig 6G). 


\section{INPP4B controls lysosomal function and response to lysosomal inhibition in leukemia cells}

To investigate a role for INPP4B in lysosomal biology in leukemia cells, we generated OCIAML2 cells with inducible-INPP4B as described above. These cells were incubated with DQ-BSAGreen and fluorescence was monitored by flow cytometry hourly, for 6 hours. INPP4B overexpressing cells proteolytically activated significantly greater levels (ANOVA $P$-value $>0.0001 \quad \mathrm{P}>$ 0.0001(Supplemental Fig 7A)) of DQ-BSA-Green fluorescence compared to controls at each time point (Fig. 7A). Similarly, experiments conducted in $\operatorname{Inpp}_{4 b^{+/+}}$and Inpp $4 b^{-/-} M L L-A F 9$ leukemias for up to 8 hours showed that Inpp $4 b^{-/}$leukemia cells had significantly lower levels of DQ-BSA-Green fluorescence throughout the time-course after incubation (ANOVA $P$-value $>0.0001$; (Fig. 7B, Supplemental Fig 7B)). Cellular uptake of fluorescence-labelled Dextran was no different in INPP4B overexpressing or control OCI-AML2 cells, nor Inpp $4 b^{+/+}$and Inpp $4 b^{-/-}$MLL-AF9 leukemia cells, indicating that endocytic uptake wsa similar and thus not responsible for the differential levels of DQBSA-Green activation (Supplemental Fig 7A-B).

Given the observations that INPP4B expression restricts leukemic differentiation and regulates lysosome function, we reasoned that lysosomes may be involved in maintaining leukemic cells in a more stem-like state. To test this hypothesis, we treated $\operatorname{Inpp}_{4 b^{+/+}}$and Inpp $4 b^{-/-} M L L-A F 9$ leukemia cells with the lysosome inhibitor Lys05 followed by LFC assays (Amaravadi and Winkler, 2012; Cechakova et al., 2019; McAfee et al., 2012). We observed that Lys05 reduced colony forming potential of all MLL-AF9 leukemia cells in a dose dependent manner. Lys05 treatment was significantly more consequential for the colony forming potential of Inpp $4 b^{+/+}$cells as compared to Inpp $4 b^{-/-}$cells (Fig. 7C). Moreover, we observed that more primitive type-I colonies were decreased with increasing doses of Lys05, whereas the more differentiated type-II colonies were increased (Fig. 7D). Together, these data show that normal lysosomal function is dependent on the presence of Inpp4b. Accordingly, the leukemic differentiating 
effects of lysosome inhibition with Lys05 is blunted in Inpp4b-deficient cells. These findings are in line with a model where INPP4B restricts leukemia differentiation through the control of lysosome function.

\section{Discussion}

Although previous studies have demonstrated that INPP4B overexpression in AML is an independent prognostic marker for poor disease outcome and a predictor of chemotherapy (Dzneladze et al., 2015; Recher, 2015; Rijal et al., 2015; Wang et al., 2016; Zhang et al., 2017), how INPP4B drives AML is poorly understood. Our studies present several lines of evidence supporting a role for INPP4B in HSC and LSC pluripotency through the regulation of lysosome biology. By investigating the consequences of Inpp4b-deficiency in both hematopoiesis and leukemogenesis in Inpp $4 b$-knockout mouse, combined with analysis of INPP4B expression in a number of human AML patient databases and cell models, we discovered that (1) INPP4B expression is enriched in primordial HSC and LSC; (2) Inpp4b-deficient leukemias have more differentiated phenotypes and exhibit increased disease latency; (3) Lysosome gene networks and function are regulated by INPP4B; (4) Disruption of lysosome functions by either INPP4B depletion or direct pharmacological inhibition leads to leukemic differentiation. Overall, by delineating INPP4B functions in AML, we have unveiled critical pathways that may underlie 'stemness' in leukemia.

$I N P P 4 B$ expression was elevated in the most pluripotent cells of hematopoietic lineages and leukemia populations, and was decreased in more differentiated populations. These data pointed to a role for INPP4B in restricting differentiation, which was confirmed in Inpp $4 b$-deficient mice where longterm repopulation of HSCs was compromised. Notably, we also observed a marked increase in cycling of Inpp4b-deficient HSCs upon myeloablation and or bone marrow reconstitution prior to exhaustion, suggesting that Inpp4b may regulate the ability of HSC to achieve quiescence after replicative stresses. Inpp $4 b$ expression mirrors the pattern of expression of genes with known HSC function over the course of 20 weeks of xenotransplant (Laurenti et al., 2015), decreasing as bone-marrow resident HSCs escape 
quiescence (up to week 4) before returning to steady-state levels as the homeostasis returns (week 20). These findings are consistent with a model where Inpp4b-deficiency limits the ability of the HSC population to remain in quiescence, instead cells experience excessive proliferation and ultimately suffer from long-term HSC exhaustion.

Analogously, Inpp $4 b^{-/-}$leukemias generated using the $M L L-A F 9$ oncogenic fusion protein, have a more differentiated AML phenotype and reduced leukemia initiating potential, further supporting a role for Inpp4b in maintaining 'stemness'. Our findings linking INPP4B to leukemia stemness identifies it as a putative therapeutic target, with potential to compromise LSC self-renewal function and consequently drive differentiation. However, given that INPP4B inhibition is not currently achievable, we sought to investigate processes downstream of INPP4B signalling with pharmacological potential. RNA sequencing of Inpp $4 b^{+/+}$and Inpp $4 b^{-/-}$leukemia combined with gene network analyses revealed that the expression of a number of key lysosomal genes including proteases and membrane transporters are under the control of Inpp4b expression. This was mirrored in AML patient data, where lysosomal gene networks were significantly associated with high INPP4B expression. Importantly, cell models of INPP4B loss and overexpression confirm that INPP4B regulates lysosome biology, including lysosomal quantity, cellular dispersion of lysosomes as well as proteolytic capacity.

Today, the lysosome is known to be a critical platform for cellular signaling that governs cell growth, division and differentiation through the integration of metabolic, nutrient and growth factor signals. Of particular importance for lysosomal functions is its intimate relationship mTORC1, the master regulator of energy and nutrient signaling (Saxton and Sabatini 2017). A direct substrate of mTORC1 is the transcription factor TFEB which regulates the transcription of a large network of lysosomal genes and thereby governs lysosomal biogenesis (Perera et al., 2019). Although INPP4B has not yet been directly linked to mTORC1 activation, its major phosphoinositide substrate $\operatorname{Ptd} \operatorname{Ins}(3,4) \mathrm{P}_{2}$, and the product if its catalysis PtdIns(3)P have been demonstrated to play critical roles in lysosomal function (Ebner et al., 2019; Inpanathan and Botelho, 2019). For instance, PtdIns(3,4) $\mathrm{P}_{2}$ can repress 
mTORC1 function on lysosomes and influence the highly motile properties of lysosomes such as anterograde (i.e. to the cell periphery) and retrograde (i.e. to the perinuclear area) transport (Bartolomeo et al., 2017; Marat et al., 2017; Munson et al., 2015). Knockdown of the PtdIns(3)P phosphatase MTMR4 has also been shown to increase lysosomal PtdIns(3)P levels and inhibit the nuclear translocation of TFEB without affecting mTORC1 (Pham et al., 2018).

Our findings highlight a novel route to LSC maintenance controlled by INPP4B and its role in lysosomal function. Further studies are required to explain the specific molecular underpinnings of INPP4B in lysosomal signalling. Nevertheless, these new data provide a basis for the utility of INPP4B as a biomarker of aggressive disease, explain its function in promoting AML aggressiveness and provide a rationale to explore INPP4B and its associated function in lysosome biology as novel strategies to target LSC and AML.

\section{Acknowledgments}

We thank all current and past members of the Salmena and Minden labs for technical assistance and critical discussions. We specifically thank Norman Iscove for technical advice; Johannes Zuber for pMSCV-MLL-AF9-IRES-mVenus plasmid; Lev Kats for invaluable advice; Robert C. Laister for encouraging deliberations and technical support. L.S. is the recipient of a Tier II Canada Research Chair (CRC) and was supported through the Human Frontier Career Development Program (HFSP) Award. This work was supported in part by funds from the Department of Pharmacology and Toxicology and Temerty Faculty of Medicine, University of Toronto and awards from Canada Foundation for Innovation (CFI- \#33505); The Natural Sciences and Engineering Research Council of Canada (NSERC-RGPIN-2015-03984); Leukemia and Lymphoma Society of Canada (LLSC-Operating Grant \#317359; Operating Grant \#422332); Acute Leukemia Translational Research Initiative through funding provided by the Ontario Institute for Cancer Research and Government of Ontario; Leukemia Research

Foundation (LRF-New Investigator Award \#169456), and Canadian Institutes for Health Research (CIHR-Operating Grant \#149032; Operating Grant \#399716) awarded to L.S. This work was supported 
in part by funds from CIHR (MOP\# 123343) awarded to J.V. Computational analyses were supported in part by funds from NSERC (\# 203475), CFI (\#225404, \#30865), Ontario Research Fund (RDI \#34876), IBM and Ian Lawson van Toch Fund awarded to I.J. R.J.B. is a recipient of a Tier II CRC, and this work was supported in part by the NSERC (RGPIN-2015-05489 and RGPIN-2020-043343).

\section{Author Contributions}

J.F.W., K.C., G.G., D.K.C.L., G.T.S., E.M.S., S.Z.X. M.H.S., M.S.G, R.H., A.R. and L.S. performed experiments. D.K.C.L., M.G., M.K., I.D. and I.J. performed bioinformatic analyses. J.V. generated Inpp $4 b$ mouse models. C.J.G, J.E.D., R.B., and M.D.M. provided critical reagents, mentorship and expertise. J.F.W. and L.S. conceived the project, planned the study and wrote the paper. L.S. secured funding for this study. All authors read and approved the text.

\section{Declaration of Interests}

All other authors declare no competing interests.

\section{Figure Titles and Legends}

Figure 1. Expression of INPP4B in hematopoietic cell lineages and Inpp4b-deficiency reduces repopulation capacity of hematopoietic progenitors. A. Relative expression of INPP $4 B$ in human hematopoietic stem and progenitor cells (data mined from (Laurenti et al., 2015)). B. Relative expression of Inpp $4 b$ in murine hematopoietic stem and progenitor cells (data mined from Lara-Astiaso et al. 2014). C. Schematic depicting INPP4B expression in the haematopoietic hierarchy (high = dark blue, low = light blue). D. Representative pseudocolour dot-plot and contour plot from immunophenotyping of the adult bone marrow niche in $\operatorname{Inpp} 4 b^{+/+}$and $\operatorname{Inpp} 4 b^{-/-}$mice $(n=6)$. E. Bone marrow stem cell fractions from age- and sex-matched adult Inpp $4 b^{+/+}$and Inpp $4 b^{-/}$mice shown as a percentage of total bone marrow monocuclear cells. F. Bone marrow progenitor fractions from age- and sex-matched adult $\operatorname{Inpp} 4 b^{+/+}$and $\operatorname{Inpp} 4 b^{-/-}$mice, shown as a percentage of total bone marrow 
monocuclear cells. G. Total colony counts from weekly serial replating of total bone marrow from ageand sex-matched $\operatorname{Inpp} 4 b^{+/+}$and $\operatorname{Inpp} 4 B^{-/}$mice (n=6, \pm S.E.M.). H. Kaplan-Meier survival analysis after serial myeloablation (5'-fluorouracil, weekly i.p.) of age- and sex-matched $\operatorname{Inpp} 4 b^{+/+}$and $\operatorname{Inpp} 4 B^{-/-}$mice $(n=10)$. I. Cell cycle analysis in sorted LSK cells, 48 hours after acute myeloablation with single 5'fluorouracil treatment, measured by intracellular Ki67 level and flow cytometry. J. Relative contribution of $\operatorname{Inpp} 4 b^{+/+}, \operatorname{Inpp} 4 b^{+/-}$and Inpp $4 b^{-/-}$long term HSCs to the regeneration of the myeloid populations in three consecutive competitive transplant experiments $(n=10, \pm$ S.E.M.). K. Relative contribution of Inpp $4 b^{+/+}, \operatorname{Inpp} 4 b^{+/}$and Inpp $4 b^{-/-}$long term HSCs to the regeneration of the total and myeloid CD45.1 (competitor) and CD45.2 (donor) populations in competitive transplant experiments at 20 weeks and 64 weeks $(n=10, \pm$ S.E.M.)

Figure 2. INPP4B expression is associated with leukemic stem cells in human AML. A. Relative expression of INPP4B from 227 AML patient-derived samples sorted based on the expression of combinations of CD34 and CD38 surface markers (Ng et al., 2016). B. Relative INPP4B expression in 278 sorted fractions characterized as $\mathrm{LSC}^{+}$and $\mathrm{LSC}^{-}$enriched by their engraftment potential into NOD/SCID mice (Ng et al., 2016). C. Gene set enrichment analysis (GSEA) of the LSC-17 gene set in INPP4B sorted AML patient samples (Ng et al., 2016) across 6 AML public patient datasets.

Figure 3. Inpp4b-deficient $M L L-A F 9$ cells have a decreased leukemogenic potential and have a more differentiated phenotype. A. Kaplan-meier survival analysis of primary Venus ${ }^{+}-M L L-A F 9$ leukemias generated from Inpp $4 b^{+/+}$and $\operatorname{Inpp} 4 B^{-/}$mouse LSK cells $(n=10)$. B. Kaplan-meier survival analysis of secondary MLL-AF9 leukemias generated by transplantation of cells from $\operatorname{Inpp} 4 b^{+/+}$and $\operatorname{Inpp}_{4 b^{-/}} M L L-A F 9$ leukemia blasts $(n=6)$. C. Limiting dilution assay (LDA) of Inpp $4 b^{+/+}$(dark blue $(n=12))$ and Inpp4b ${ }^{-/}$MLL-AF9 (light blue $\left.(n=12)\right)$ leukemia cells $(1000,250,100$, and 25 cells) into host wild-type C57BL/6 mice. D. Representative image of type-I and type-II colonies from secondary 
MLL-AF9 leukemia cells (left). Total and percentage colony counts in LFC assays from Inpp $4 b^{+/+}$and Inpp $4 B^{-/-}$murine $M L L-A F 9$ leukemia blasts ( $n=8, \pm$ S.E.M.; right). E. Representative images of undifferentiated and differentiated blasts from $\operatorname{Inpp}_{4 b^{+/+}}$and $\operatorname{Inpp} 4 b^{-/-} M L L-A F 9$ terminal leukemias $\left(n=3, \pm\right.$ S.E.M.; left). Proportion of undifferentiated and differentiated leukemic blasts from Inpp $4 b^{+/+}$ (right). F. Contour plots (10\% probability) show CD11b versus Gr1 expression on live singlets from Inpp $4 b^{+/+}$versus Inpp $4 b^{-/} M L L-A F 9$ leukemia samples compared to WT BM cells (representative of $\mathrm{n}=3$ /group). Scatter graph shows the $\% \mathrm{CD} 1 \mathrm{~b}^{+} \mathrm{Gr}^{+}$cells in each AML type. Two-tailed t-test with Welch's correction, $\mathrm{P}=0.001$. G. Top: Contour plots (tshow CD16/32 versus CD117 expression on $\mathrm{CD}_{11 \mathrm{~b}^{+}} \mathrm{Gr}^{-}$cells from representative $\operatorname{Inpp}_{4 b^{+/+}}$and Inpp $4 b^{-/-} M L L-A F 9$ leukemia samples. Scatter graph shows the \%CD16/32 ${ }^{+} \mathrm{CD} 117^{+}$cells in each type of AML. Two-tailed t-test with Welch's correction, $\mathrm{P}=0.1726$. Bottom: Heatmap visualization of the median metal intensity (MMI) of the indicated markers on $\mathrm{CD}_{11 \mathrm{~b}^{+}} \mathrm{Gr}^{-}$cells from each type of AML. Values for each marker were calculated as $\operatorname{arcinsh}(\operatorname{MMI}(x) / \square$ scale_argument)- $\square \operatorname{arcsinh}($ control /scale_argument) where $x$ is the sample value, control is the lowest value for the marker across all samples, and scale argument=5. Multiple unpaired t-tests with Welch's correction. Multiple comparisons corrected by False Discovery Rate using the 2-stage step-up method of Benjamini, Krieger and Yekiuteli. *, Q=0.03 for each comparison shown.

Figure 4. Inpp4b expression is associated with lysosomal gene sets. A.Top 20 enriched KEGG gene sets in Inpp $4 b^{+/+}$and Inpp $4 b^{-/}$MLL-AF9 leukemia blast cells determined using GSEA. B. Top 20 pathways from InnateDB Gene Ontology (GO) over-representation analysis (ORA) of all genes with significantly altered expression in Inpp $4 b^{+/+}$and Inpp $4 b^{-/-}$MLL-AF9 leukemia blast cells (Breuer et al., 2013). C. Heat map of differentially expressed KEGG Lysosome genes from Inpp $4 b^{+/+}$and $\operatorname{Inpp}_{4} b^{-/}$ MLL-AF9 leukemia blast cells (upregulated in red, downregulated in blue). D. STRING-db analysis of differential expression of KEGG Lysosome genes from Inpp $4 b^{+/+}$and Inpp $4 b^{-/} M L L-A F 9$ leukemia 
blast cells. E-F. Gene set expression analysis (GSEA) for Lysosome pathway genes in $\operatorname{Inpp} 4 b^{-/-} M L L-$ AF9 leukemia blast cells with E. KEGG Lysosome gene set; F. Lysosomal proteins gene set ((Sardiello et al., 2009)); G. LSC17 signature gene set ((Mootha et al., 2003; Ng et al., 2016; Subramanian et al., 2005)).

Figure 5: A Lysosome gene set is enriched in patients with low levels of INPP4B. A-B. Scatter plot of the top 20 enriched KEGG gene sets in INPP4B-low patients from the A. TCGA and B. Verhaak dataset. C-F. GSEA plots for the KEGG lysosome gene set in INPP4B-low patients from the C. TCGA AML; D. Verhaak AML; E. Valk AML; F. Wouters AML dataset. G. Venn diagram illustrating the genes from the KEGG lysosome gene set that contribute to the leading edge subset in the four AML datasets. H. Table of the common core enriched lysosome genes among the four AML datasets.

Figure 6: INPP4B regulates lysosomal biology. A. Representative immunoblot and micrograph of INPP4B expression in U2OS cells upon INPP4B induction with doxycycline (100mg/mL). B. Representative immunoblot and micrograph of INPP4B expression in U2OS upon treatment with siRNA targeting INPP4B or control siRNA. C. Representative micrograph of LAMP1 dispersion across perinuclear shells (1-4) in U2OS cells. D. Quantification of LAMP1 puncta in perinuclear shells (1-4) in U2OS cells upon INPP4B induction or siRNA-mediated knockdown of INPP4B. E. Quantification of LAMP1 puncta and total cellular LAMP1 staining intensity in U2OS upon treatment with small interfering RNA targeting INPP4B or a non-specific control. F-G. Representative micrograph and

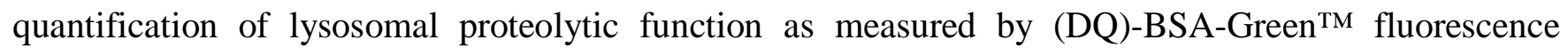
relative to Dextran-Red in U2OS cells upon INPP4B induction with doxycycline or siRNA-mediated knockdown of INPP4B. 
Figure 7: INPP4B regulates lysosomal biology in leukemia cells. A. Inducible-INPP4B OCI-AML2 cells were treated without or with doxycycline $(100 \mathrm{mg} / \mathrm{mL})$ and incubated with DQ-BSA-Green ${ }^{\mathrm{TM}}$ and fluorescence was monitored by flow cytometry hourly, for up to 6 hours. B. Inpp $4 b^{+/+}$and Inpp $4 B^{-/-}$ MLL-AF9 leukemia blasts were incubated with DQ-BSA-Green ${ }^{\mathrm{TM}}$ and fluorescence was monitored by flow cytometry hourly, for up to 8 hours. C. Inpp $4 b^{+/+}$and $\operatorname{Inpp} 4 B^{-/-} M L L-A F 9$ leukemia blasts were plated in the CFC assay in the presence of Lys05 for 7 days. Violin/dot plots represent normalized colony counts. D. Inpp $4 b^{+/+} M L L-A F 9$ leukemia blasts were plated in the LFC assay in the presence of Lys05 for 5 days. 


\section{Key Resources Table}

\begin{tabular}{|c|c|c|}
\hline REAGENT or RESOURCE & SOURCE & IDENTIFIER \\
\hline \multicolumn{3}{|l|}{ Antibodies } \\
\hline Anti-mouse lineage Cocktail & Biolegend & 133313 \\
\hline anti-mouse CD45.1 PE & Biolegend & 110708 \\
\hline anti-mouse CD45.2 FITC & Biolegend & 109806 \\
\hline $\begin{array}{l}\text { anti-mouse Ly-6A/E } \\
\text { PE/Cyanine } 7\end{array}$ & Biolegend & 108114 \\
\hline anti-mouse CD150 PE & Biolegend & 115904 \\
\hline anti-mouse CD48 BV $421^{\mathrm{TM}}$ & Biolegend & 103427 \\
\hline anti-mouse CD117 APC & Biolegend & 105812 \\
\hline anti-mouse CD16/32 BV 510 & Biolegend & 101333 \\
\hline anti-mouse CD34 & BD Pharmingen $^{\mathrm{TM}}$ & 560238 \\
\hline $\begin{array}{l}\text { anti-mouse/human CD11b } \\
\text { PE/Cyanine7 }\end{array}$ & Biolegend & 101216 \\
\hline $\begin{array}{l}\text { anti-mouse Ly-6G/Ly-6C } \\
\text { PE/Cyanine5 }\end{array}$ & Biolegend & 108410 \\
\hline Anti mouse EPCR PE & Biolegend & 141503 \\
\hline Anti mouse Ki67 FITC & Biolegend & 652410 \\
\hline $\begin{array}{l}\text { Anti mouse Gr1, Ly6G/C; } 141 \\
\text { Pr; Clone RB6-8C5 }\end{array}$ & Guidos lab, in house & \\
\hline $\begin{array}{l}\text { Anti mouse CD11b, Itgam; } 148 \\
\text { Nd; M1/70 }\end{array}$ & Guidos lab, in house & \\
\hline $\begin{array}{l}\text { Anti mouse CD19; } 149 \text { Sm; } \\
\text { Clone 1D3 }\end{array}$ & Guidos lab, in house & \\
\hline $\begin{array}{l}\text { Anti mouse CD24; } 150 \mathrm{Nd} \text {; } \\
\text { Clone M1/69 }\end{array}$ & Guidos lab, in house & \\
\hline $\begin{array}{l}\text { Anti mouse CD3e; } 152 \mathrm{Sm} \text {; } \\
\text { Clone 145-2C11 }\end{array}$ & Guidos lab, in house & \\
\hline Anti mouse TER-119, Ly76; & Guidos lab, in house & \\
\hline
\end{tabular}




\begin{tabular}{|c|c|c|}
\hline 154 Sm; Clone TER-119 & & \\
\hline $\begin{array}{l}\text { Anti mouse CD16/32; } 155 \mathrm{Gd} \text {; } \\
\text { Clone } 2.4 \mathrm{G} 2\end{array}$ & Guidos lab, in house & \\
\hline $\begin{array}{l}\text { Anti mouse CD } 45 ; 156 \mathrm{Gd} \text {; } \\
\text { Clone } 30-\mathrm{G} 12\end{array}$ & Guidos lab, in house & \\
\hline $\begin{array}{l}\text { Anti mouse Flt3, CD135; } 162 \\
\text { Dy; Clone A2F10.1 }\end{array}$ & Guidos lab, in house & \\
\hline $\begin{array}{l}\text { Anti mouse Sca1, Ly-6A/E; } 164 \\
\text { Dy; Clone E13-161.7 }\end{array}$ & Guidos lab, in house & \\
\hline $\begin{array}{l}\text { Anti mouse CD117, ckit; } 166 \\
\text { Er; Clone 2B8 }\end{array}$ & Guidos lab, in house & \\
\hline $\begin{array}{l}\text { Anti mouse CD150, SLAM; } 167 \\
\text { Er; Clone TC15-12F12.2 }\end{array}$ & Guidos lab, in house & \\
\hline $\begin{array}{l}\text { Anti mouse CD44; } 171 \mathrm{Yb} \text {; } \\
\text { Clone IM7 }\end{array}$ & Guidos lab, in house & \\
\hline $\begin{array}{l}\text { Anti mouse MHC class II; } 174 \\
\text { Yb; Clone M5/114.15.2 }\end{array}$ & Guidos lab, in house & \\
\hline $\begin{array}{l}\text { Anti mouse B220; } 176 \mathrm{Yb} \text {; } \\
\text { Clone RA3-6B2 }\end{array}$ & Guidos lab, in house & \\
\hline Anti human LAMP1 & Cell Signaling Technology & $9091 S$ \\
\hline Anti human INPP4B & Cell Signaling Technology & $14543 \mathrm{~S}$ \\
\hline Anti HA-tag & Cell Signaling Technology & $3724 S$ \\
\hline \multicolumn{3}{|c|}{ Chemicals, Peptides, Recombinant Proteins } \\
\hline Ammonium Chloride & Stem Cell Technologies & 07850 \\
\hline Mouse Recombinant IL-3 & Stem Cell Technologies & 78042 \\
\hline Mouse Recombinant IL-6 & Stem Cell Technologies & 78052 \\
\hline Mouse Recombinant SCF & Stem Cell Technologies & 78064 \\
\hline MethoCult ${ }^{\mathrm{TM}}$ GF M3434 & Stem Cell Technologies & 03444 \\
\hline 5' Fluorouracil & Gift from Mark Minden & N/A \\
\hline
\end{tabular}




\begin{tabular}{|c|c|c|}
\hline Cytarabine & Gift from Mark Minden & N/A \\
\hline \multicolumn{3}{|l|}{ Critical Commercial Assays } \\
\hline Mouse Cell Depletion Kit & Milteny Biotec & $130-104-694$ \\
\hline LS Columns & Milteny Biotec & $130-042-401$ \\
\hline RNeasy Mini Kit & Qiagen & 74104 \\
\hline $\mathrm{DQ}^{\mathrm{TM}}$ Green BSA & Invitrogen/Thermo Fisher & D12050 \\
\hline $\mathrm{DQ}^{\mathrm{TM}} \operatorname{Red} \mathrm{BSA}$ & Invitrogen/Thermo Fisher & D12051 \\
\hline Dextran, Alexa Fluor ${ }^{\mathrm{TM}} 647$ & Invitrogen/Thermo Fisher & D22914 \\
\hline Lucifer Yellow CH & Invitrogen/Thermo Fisher & $\mathrm{L} 453$ \\
\hline $\begin{array}{l}\text { FuGENE® HD Transfection } \\
\text { Reagent }\end{array}$ & Promega & E2311 \\
\hline \multicolumn{3}{|c|}{ Experimental Models: Organisms/Strains } \\
\hline C57BL/6 & Charles River Canada & Strain Code: 027 \\
\hline $\begin{array}{l}\text { B6.SJL-PtprcaPepcb/BoyJ } \\
\text { (CD45.1) mice }\end{array}$ & The Jackson Laboratory & Stock No: 002014 \\
\hline Inpp $4 b^{-/-}$mice & Jean Vacher, IRCM & Ferron et al. 2011 \\
\hline
\end{tabular}

\section{Resource Availability}

Lead Contact: Further information and requests for resources and reagents should be directed to and will be fulfilled by the Lead Contact, Leonardo Salmena (leonardo.salmena@utoronto.ca).

Materials Availability: This study did not generate new unique reagents.

Data and Code Availability: The RNA sequencing data will be made available at Gene Expression Omnibus. All other datasets generated in this study are available within the paper or from the lead contact upon reasonable request. 


\section{Experimental Model and Subject Details}

Mice. C57BL/6 wild-type mice were bred and housed at the University Health Network/Princess Margaret Cancer Center and the Division of Comparative Medicine Facility at the University of Toronto.

B6.SJL-Ptprca Pepcb/BoyJ (CD45.1) mice were purchased from The Jackson Laboratory (Bar Harbor, Maine, USA). All animal experiments were performed in accordance with national and institutional guidelines approved by the Canadian Counsel on Animal Care and approved by the University Health

Network Animal Care Committee and the Division of Comparative Medicine at the University of Toronto. Inpp $4 b^{-/-}$mice kindly provided by Jean Vacher (Institut de Recherches Cliniques de Montréal) and were genotyped as previously described (Ferron et al., 2011).

\section{Method Details}

Immunophenotyping of Inpp $4 b^{-/}$mice. Total bone marrow was extracted from the hind legs of $\operatorname{Inpp} 4 b^{+/+}, \operatorname{Inpp} 4 b^{+/-}$and Inpp $4 b^{-/}$mice by crushing into ice-cold Iscove's Modified Dulbecco's Medium (IMDM) medium. RBC lysis was performed using standard ammonium chloride procedure. Total bone marrow mononuclear cell (MNCs) were stained for surface marker expression in PBS. 2mM EDTA, 2\% FBS for 90 minutes at $4^{\circ} \mathrm{C}$. The following antibody cocktails were used: anti-mouse CD3 (145-2C11), anti-mouse CD45R/B220 (RA3-6B2, anti-mouse CD11b (M1/70), anti-mouse Erythroid marker (TER119), anti-mouse Ly-6G (RB6-8C5), anti-mouse CD34 (RAM34), anti-mouse Flk2 (A2F10), anti-mouse c-kit (2B8), anti-mouse Sca1 (D7), anti-mouse CD150 (TC15-12F12.2), anti-mouse CD48 (HM48-1), anti-mouse CD201/EPCR (eBio1560). All antibodies were obtained from eBiosciences, BD Pharmingen or Biolegend. Cells were analysed on a LSR Fortessa X20 (BD Biosciences). 
MLL-AF9 AML Models. MLL-AF9 expressing cells were generated by retroviral transduction of murine bone marrow progenitors. Briefly, bone marrow was flushed from the long bones of 10-week old Inpp $4 b^{+/+}$and Inpp $4 b^{-/-}$mice. From these total bone marrow preparations, $2 \times 10^{6}$ LSK cells were sorted and grown for 24 hours in IMDM supplemented with $20 \mathrm{ng} / \mathrm{ml} \mathrm{SCF}, 10 \mathrm{ng} / \mathrm{ml} \mathrm{IL-6}$ and $10 \mathrm{ng} / \mathrm{ml}$ IL-3 (Gibco). Inpp $4 b^{+/+}$and Inpp $4 b^{-/}$LSK were each retrovirally transduced with pMSCV-MLL-AF9-IRESmVenus. 24 hours post retroviral transduction, $2 \times 10^{5}$ Venus $^{+}$cells were transplanted to sub-lethally irradiated (4.5Gy) donor C57BL/6 mice to generate primary leukemias (Zuber et al., 2009). Subsequent transplantation of $\mathrm{mVenus}^{+}$cells from mice with terminal leukemias were designated as secondary leukemia assays. In vitro assays were performed in IMDM medium with $2 \%$ methylcellulose, supplemented with $20 \mathrm{ng} / \mathrm{ml} \mathrm{SCF}, 10 \mathrm{ng} / \mathrm{ml}$ IL-6 and $10 \mathrm{ng} / \mathrm{ml}$ IL-3 (Stem Cell Technologies). Cytological analysis of blood and bone marrow smears from Inpp $4 b^{+/+}$and $\operatorname{Inpp} 4 b^{-/-}$leukemic mice was done by classical wright-giemsa staining (Sigma). For limiting dilution transplantation assays (LDA), $M L L-A F 9$ leukemia cells were injected at defined doses (equivalent to 10, 25, 50, 100, and 1000 blast cells) into 8-week-old female C57BL/6 mice. LSC frequency was estimated using the online tool ELDA (http://bioinf.wehi.edu.au/software/elda/index.html) ((Hu and Smyth, 2009).

Competitive Transplant of Inpp4 $\boldsymbol{b}^{-/-}$bone marrow. Lethally irradiated C57BL/6-CD45.1 congenic mice were reconstituted with bone marrow MNCs from age- and sex-matched Inpp $4 b^{+/+}$or Inpp $4 b^{-/-}$ mice (CD45.2), in competition with bone marrow MNCs from C57BL/6-CD45.1 mice. Competitive transplants were carried out in a 1:1 ratio, with $1 \times 10^{6}$ cells from each combined prior to injection. Reconstitution of donor-derived cells (CD45.2) was monitored by staining blood cells with mAbs against CD45.2, CD45.1, Mac-1, and Gr-1. For the serial transplantation analysis, bone marrow cells (1 $\times 10^{6}$ ) were obtained from recipient mice at 20 weeks post-transplantation and transplanted into a 
second set of lethally irradiated mice. The ratio of 1:1, Cd45.1:CD45.2 was re-established prior to secondary transplant. Subsequent transplants were performed in the same manner.

Gene expression data. Genome-wide expression data from The Cancer Genome Atlas (TCGA)-LAML dataset was downloaded from the ICGC database (https://icgc.org/). Normalized microarray data from the Herold (GSE37642-GPL570), Klein (GSE15434), Metzeler (GSE12417-GPL570), Valk (GSE1159), Verhaak (GSE6891), Wouters (GSE14468), Laurenti (GSE42414, GPL14951), Notta (GSE76234), Novershtern (GSE24759), Pietras (GSE68529), Rapin (GSE42519) and Lara-Asiato (GSE60101) datasets were downloaded from the GEO database (https://www.ncbi.nlm.nih.gov/geo/).

In vitro CFU Assays of Inpp4b ${ }^{-/}$bone marrow. To determine the re-plating ability of HSPCs $\operatorname{Inpp} 4 b^{+/+}$and Inpp $4 b^{-/-}$we plated bone marrow from age- and sex-matched mice in Methocult GF M3434 (Stem Cell Technologies) according to the manufacturer's instructions. Briefly, total mouse bone marrow MNCs were flushed from long bones into IMDM medium. RBCs were lysed in ammonium chloride solution and cells were resuspended in IMDM. 2 × $10^{4}$ cells were plated onto 35-mm dishes and incubated at $37^{\circ} \mathrm{C}, 5 \% \mathrm{CO}_{2}$ for 7-days. After 7-days colonies were counted and scored. 2 × $10^{4}$ cells were re-plated onto fresh methocult every 7 days and counted, until exhaustion.

5'-FU Treatment. Age- and sex-matched $\operatorname{Inpp} 4 b^{+/+}$or Inpp $4 b^{-/-}$mice were treated with 5'-FU $(150 \mathrm{mg} / \mathrm{kg}$ in saline, i.p.) or saline control, once weekly. Survival analysis was performed by the Kaplan-Meier method. Necropsies were performed and extracted spleens were weighed. Separately, LSK cells were sorted 48-hours after treatment with a single dose of 5'-FU from age- and sex-matched Inpp $4 b^{+/+}$and Inpp $4 b^{-/-}$mice. These LSK cells were then fixed and cycling was assessed by flow cytometry measurement of Ki67 levels. 
Blood Analysis. Total blood counts for Age- and sex-matched Inpp $4 b^{+/+}$and Inpp $4 b^{-/-}$mice $(n=10)$ were performed using a VetScan HM5 (Abaxis) by the Division of Comparative Medicine (University of Toronto).

Mass Cytometry. Purified mAbs were conjugated with heavy metals by the Flow and Mass Cytometry Facility, The Hospital for Sick Children. Murine AML blasts were counted and $2 \times 10^{6}$ cells for each sample were stained for cell surface markers in staining media (PBS containing 1\% BSA and $0.02 \%$ NaN3) for 30 minutes at 4C. Cells were washed with protein-free PBS, stained with $1 \mathrm{mmol} / \mathrm{L}$ cisplatin for 5 minutes at room temperature, fixed using the transcription factor buffer set (BD Biosciences) followed by intracellular staining for 60 minutes at 4C. Cells were washed with staining media and stained with $100 \mathrm{nmol} / \mathrm{L}$ iridium-labeled DNA-intercalator (Fluidigm) in PBS containing 0.3\% saponin and $1.6 \%$ formaldehyde at $4 \mathrm{C}$ for up to 48 hours. Cells were washed twice with deionized water prior to adding EQ normalization beads containing Ce140, Eu151, Eu153, Ho165, and Lu175 (Fluidigm) and acquiring on a Helios mass cytometer by The Flow and Mass Cytometry Facility, The Hospital for Sick Children. After normalizing and randomizing values near zero using the Helios software, FCS files were uploaded to Cytobank for analysis.

RNA sequencing. $\operatorname{Inpp} 4 b^{+/+}$or Inpp $4 b^{-/-} M L L-A F 9$ leukemias (3 in total for each genotype) were sorted for Venus+ before isolation of total cellular RNA with an RNeasy isolation kit (Qiagen). A Bioanalyzer 2100 (Agilent) was used for quality control and quantification. Illumina MouseRef-8 v2.0 Expression BeadChip kits were used for genome-wide expression profiling according to standard protocols at The Centre for Applied Genomics core facility at the Hospital for Sick Children. R 
Bioconductor 2.13.0 software was used for data processing and other statistical analyses. Raw signals from 25,697 probes were pre-processed for background subtraction, quantile normalization and $\log 2$ transformation before the use of moderated t-tests from the Bioconductor software package Limma (linear models for microarray data). Empirical Bayes smoothing was applied to the standard errors. Paired t-tests were used for the identification of differentially expressed genes expression in each genotype subset, and the false-discovery rate (FDR) was estimated with the Benjamini-Hochberg method to correct for multiple testing. Pearson correlations showed that technical replicates had very high correlations between chips. For genes represented by multiple probe sets on the array, we selected the ones with the highest ANOVA F-statistics (lowest FDR-adjusted q value).

\section{Bioinformatics}

Gene Set Enrichment Analysis (GSEA). GSEA was performed on the datasets using GSEA v.4.0.3 provided by the Broad Institute (http://software.broadinstitute.org/gsea/downloads.jsp). Samples were rank ordered and split by INPP4B status, $25 \%$ high/75\% low (human) or Inpp $4 b^{+/+} / \operatorname{Inpp} 4 b^{-/-}$(mouse). Enriched gene sets were identified by 1,000 phenotype permutations in the human datasets, and 1,000 gene set permutations in the mouse dataset. Gene sets with a nominal $p$--value $<0.05$ were considered significantly enriched. The curated KEGG $\quad$ (CP: $\quad$ KEGG) and BROWN_MYLEOID_CELL_DEVELOPMENT_UP gene set were obtained from MSigDB Collections (http://software.broadinstitute.org/gsea/msigdb/collections.jsp) and the LSC17 and Lysosomal Proteins gene sets were generated from the indicated publication (Ng et al., 2016; Palmieri et al., 2011).

Gene Ontology (GO). GO analysis was performed using the InnateDB database (https://www.innatedb.ca/; Breuer et al., 2013).

Gene Network Analysis. The lysosome interacting genes were passed through String-db.v.11 (Szklarczyk et al. 2019). and the combined binding score for each protein-protein interaction was 
obtained. This output, which is experimentally determined and annotated, connects the protein nodes. The absolute log fold change from the RNAseq is used to size the nodes and the colour corresponds to the $-\log _{10}$ (Pvalue). The interaction map was generated by 'ForceAtlas2' in Gephi.v.0.9.2. (Jacomy et al. $\underline{2014)}$

\section{Immunofluorescence and DQ-BSA assays in U2OS cells}

For LAMP1 immunofluorescence, U2OS cells were fixed with ice-cold methanol (100\%) for 5 min at 20 C. Cells were then washed with PBS and incubated for $1 \mathrm{~h}$ in blocking buffer (10\% FBS, $3 \%$ BSA in PBS) before the addition of antibody. Anti-LAMP1 rabbit XP® monoclonal antibodies $(1: 100$, Clone D2D11, Cell Signaling) were added to cells in blocking buffer and incubated for $1 \mathrm{~h}$ at room temperature. Secondary anti-rabbit antibodies were then added to cells at a 1:1000 dilution in blocking buffer and incubated for $1 \mathrm{~h}$ at room temperature. After the indicated time, cells were stained with 1 $\mu \mathrm{g} / \mathrm{mL}$ DAPI (Roche Biochemicals). Cells were washed three times with $0.3 \%$ BSA in PBS between each step, and washed a final time before mounting coverslips with Dako fluorescent mounting medium. Z-stacks of cells were acquired at $0.3 \mu \mathrm{m}$ interval steps with a Quorum DisKovery Spinning Disc confocal microscope system equipped with a Leica DMi8 microscope connected to Andor Zyla 4.2 Megapixel sCMOS camera and controlled by Quorum Wave FX Powered by MetaMorph software (Quorum Technologies, Guelph, ON). LAMP1 puncta, fluorescence intensity, and positioning were quantified using ImageJ software. For lysosome positioning, each cell was outlined manually to generate a region of interest (shell), and the same shell was reduced in size by 15-pixel iterations to produce four shells in total per cell. In all cases, thresholding for lysosomal signal was conducted manually followed by particle detection analysis (particles with a minimum area of 2 pixel) and fluorescence intensity for each shell, and normalized to shell area. Images obtained from randomly selected 60 cells from 3 independent experiments. To measure the proteolytic capacity of lysosomes, cells were treated with 10 $\mu \mathrm{g} / \mathrm{mL}$ DQ Green BSA (Invitrogen) and $2 \mu \mathrm{M}$ fixable Alexa-647-conjugated $10 \mathrm{kDa}$ dextran 
(Invitrogen) for $6 \mathrm{~h}$, followed by a $1 \mathrm{~h}$ chase in label-free media. Following a chase period, cells were fixed in 4\% PFA for 20 min and imaged. DQ-BSA and Alexa-647-dextran images were acquired using an Olympus IX83 microscope system with a Hamamatsu ORCA-Flash4.0 digital camera controlled by CellSens Dimensions software (Olympus Canada Inc., Richmond Hill, ON). Images obtained from randomly selected 60 cells from 3 independent experiments were quantified by using ImageJ. In all cases, thresholding for DQ Green BSA and dextran signals were conducted manually followed by fluorescence intensity for the whole cell. The mean intensity of the whole cell was obtained by drawing an area covering the whole cell. Subsequently fluorescent intensities were quantified by normalizing the DQ-BSA fluorescence signal against the Alexa-647 signal after background correction.

DQ-BSA in $M L L-A F 9$ cells. $M L L-A F 9$ Inpp4b+/+ and Inpp4b-/- cells were cultured for $48 \mathrm{~h}$ and AML2-TETON-INPP4B cells were treated with doxycycline $(100 \mathrm{ng} / \mathrm{mL})$ prior to lysosome labelling. Cells were incubated with 10ug/mL DQ Red BSA (Invitrogen) or 2uM Alexa-647-conjugated 10kDa dextran (Invitrogen) in complete media for $1 \mathrm{~h}$ to $6 \mathrm{~h}$ at $37^{\circ} \mathrm{C}$. At each time point, cells were washed twice with PBS and subsequently analyzed for whole cell fluorescence with Beckman Coulter Cytoflex flow cytometer. A total of 60,000 events were collected per sample per time point using the APC channel. Non-labeled cells were used as control for determining background signal and as time point 0 .

Lysosome labelling. U2OS lysosomes were labelled by incubating with Lucifer yellow $2.5 \mathrm{mg} / \mathrm{ml}$ (Thermo Fisher Scientific, Mississauga, $\mathrm{ON}$ ) for $4 \mathrm{~h}$ in complete media at $37^{\circ} \mathrm{C}$ and $5 \% \mathrm{CO}_{2}$. Cells were washed twice with phosphate-buffered saline (PBS) and supplemented with complete media for $1 \mathrm{~h}$. 
Spinning disc confocal microscopy. Imaging performed through spinning disc confocal microscopy with an Olympus IX81 inverted microscope equipped with a Hamamatsu C9100-13 EMCCD camera controlled by Volocity 6.3.0 (PerkinElmer, Bolton, ON). Time lapse live imaging was performed at single z-focal planes using complete media in a $5 \% \mathrm{CO}_{2}$ chamber at $37^{\circ} \mathrm{C}$.

Image analysis. To analyze the lysosome associated to cytosol ratio of INPP4B-mCherry, images imported to ImageJ, and 5-pixel wide lines measuring 40-pixel in length were designated to nucleusexcluded areas of the cell. Intensity plot profiles acquired and exported to excel spreadsheet, and values assorted according to intensity. Ratio obtained of highest 10 pixels over lowest 10 pixels $\left(\mathrm{F}_{\mathrm{H}} / \mathrm{F}_{\mathrm{L}}\right.$ fluorescent ratio) where intensity signal-values representing cytosolic distribution was expected to have a ratio value of approximately 1 .

Lentivirus production and generating TMEM192-3xHA stable cells. HEK293T cells were transfected with FuGene HD (Promega, Madison, WI) for pLJC5-TMEM192-3xHA (addgene) in combination with VSV-G and psPAX2 packaging plasmids to generate lentivirus. Media was replaced $24 \mathrm{~h}$ post transfection with fresh complete media for $48 \mathrm{~h}$. Virus containing media was collected, and cells were supplemented with fresh complete media for $24 \mathrm{~h}$ followed by collection of virus containing media. Virus containing media was centrifuged at $1000 \mathrm{rpm}$ for $3 \mathrm{~min}$ to remove cells and stored at $80^{\circ} \mathrm{C}$. To generate U2OS cells stably expressing TMEM192-3xHA, 1 million U2OS cells plated in 10 $\mathrm{cm}$ plates were overlayed with two $24 \mathrm{~h}$ rounds of $10 \mathrm{ml}$ virus containing media supplemented with 8 $\mu \mathrm{g} / \mathrm{ml}$ protamine sulfate. Virus containing media was replaced with fresh complete media for $24 \mathrm{~h}$ followed by $48 \mathrm{~h}$ selection with $2 \mu \mathrm{g} / \mathrm{ml}$ puromycin. 
Lysosome immunoprecipitation. $~ 30$ million cells plated in $15 \mathrm{~cm}$ plates were washed with PBS and collected through trypsinization. Cells were washed twice at $1000 \mathrm{x}$ g for 2 min with $1 \mathrm{ml}$ of KPBS (136 $\mathrm{mM} \mathrm{KCl}, 10 \mathrm{mM} \mathrm{KH} \mathrm{PO}_{4}, \mathrm{pH}$ adjusted to 7.25 with $\mathrm{KOH}$ ). Cell pellets resuspended and $20 \mu \mathrm{l}$ collected, lysed with RIPA buffer and stored at $-20^{\circ} \mathrm{C}$ as input. The remaining $980 \mu \mathrm{l}$ of cells were homogenized with 25 strokes of a $7 \mathrm{ml}$ homogenizer. The homogenate was centrifuged $1000 \mathrm{x} \mathrm{g}$ for 2 min. The supernatant containing organelles was collected and incubated with $100 \mu$ of KPBS prewashed anti-HA magnetic beads for $15 \mathrm{~min}$ on a gentle rotating shaker. The immunoprecipitates were washed four times with KPBS buffer on DynaMag-2 Spin Magnet (Thermo Fisher Scientific, Mississauga, ON). The immunoprecipitates and input were subjected to immunoblotting with the following primary antibodies: Rabbit monoclonal antibody for INPP4B (1:1000, 14543, Cell Signaling) and HA (1:1000, 3724, Cell Signaling), Rabbit polyclonal antibody for VAPB (1:2000, HPA013144, Sigma, Oakville, ON), and Rabbit monoclonal antibody for LAMP1 (1:1000, 9091, Cell Signaling).

\section{Quantification and Statistical Analysis}

Errors bars show standard error of the mean (SEM). Data were analyzed with a two-tailed Student t-test for comparison of the means of two groups and by one-way ANOVA. P values of less than 0.05 were considered statistically significant. No randomization of mice or 'blinding' of researchers to sample identity was used during the analyses. Sample sizes were not predetermined on the basis of expected effect size, but rough estimations were made on the basis of pilot experiments and measurements. No data exclusion was applied. 


\section{Supplemental Information Titles and Legends}

Supplementary Figure 1. A. Relative expression of INPP4B in human hematopoietic stem and progenitor cells (data mined from (Jung et al., 2015; Laurenti et al., 2013, 2015; Lechman et al., 2016; Notta et al., 2016; Novershtern et al., 2011; Rapin et al., 2014)) B. Relative expression of Inpp4b in mouse hematopoietic stem and progenitor cells (data mined from Pietras et al., 2015). C. Total bone marrow cellularity from age- and sex-matched $\operatorname{Inpp} 4 b^{+/+}$and $\operatorname{Inpp} 4 B^{-/-}$mice $(n=10, \pm$ S.E.M.). D. Total peripheral blood counts from age- and sex-matched adult Inpp $4 b^{+/+}$and Inpp $4 b^{-/-}$mice (normalized to control. $n=10$, mean \pm SEM). E. Flow cytometry gating strategy for immunophenotyping of HSPCs in $\operatorname{Inpp} 4 b^{+/+}$and $\operatorname{Inpp} 4 B^{-/-}$mice. F. Representative contour plot of EPCR expression in $\operatorname{Inpp} 4 b^{+/+}$and Inpp $4 B^{-/}$LT-HSCs. G. Individual colony counts from weekly serial replating of total bone marrow from age- and sex-matched $\operatorname{Inpp} 4 b^{+/+}$and $\operatorname{Inpp} 4 B^{-/}$mice $(n=6, \pm$ S.E.M.). H. Flow cytometry gating strategy for CD45.2 (test cells) and CD45.1 (competitor cells) percentages in myeloid cells in long term competitive transplant assays.

Supplementary Figure 2. Heatmaps of LSC-17 gene expression in the AML patient datasets (Cancer Genome Atlas Research Network et al., 2013; Herold et al., 2018; Klein et al., 2009; Metzeler et al., 2008; Palmieri et al., 2011; Valk et al., 2004; Verhaak et al., 2009; Wouters et al., 2009), ranked by INPP4B expression (INPP4B-high quartile highlighted dark blue).

Supplementary Figure 3. A. Gating strategy for generation of $M L L-A F 9$ murine leukemias from ageand sex-matched adult Inpp $4 b^{+/+}$and Inpp $4 b^{-/-}$mice, expressing both Gr-1 and Mac-1 surface markers.

Supplementary Figure 4. A. Scatter plot of TPM (transcript per million) values from genes with significantly altered expression in $\operatorname{Inpp} 4 b^{+/+}$and $\operatorname{Inpp} 4 b^{-/-} M L L-A F 9$ leukemia blast cells $(n=3$, FDR < 0.01). B. Volcano plot of all genes from $\operatorname{Inpp}_{4} b^{+/+}$and $\operatorname{Inpp} 4 b^{-/-} M L L-A F 9$ leukemia blast cells $(n=3$, 
FDR < 0.01). C. GSEA enrichment shows a positive correlation between Inpp $4 b^{-/} M L L-A F 9$ leukemia blast cells and myeloid cell development genes. D. GSEA enrichment shows a positive correlation between $\operatorname{Inpp} 4 b^{-/-} M L L-A F 9$ leukemia blast cells and osteoclast differentiation genes.

Supplementary Figure 5. A-D. GSEA and heatmaps between INPP4B expression and the KEGG lysosomal protein gene set across four AML public patient datasets. E-H. GSEA and heatmaps between INPP4B expression and the TFEB-regulated Lysosomal Proteins. AML patient datasets, ranked by INPP4B expression (INPP4B-high quartile highlighted yellow) also shown (Cancer Genome Atlas Research Network et al., 2013; Palmieri et al., 2011; Valk et al., 2004; Verhaak et al., 2009; Wouters et al., 2009)

Supplementary Figure 6. A. Quantification of LAMP1 puncta and total cellular LAMP1 staining intensity in U2OS cells upon doxycycline induced INPP4B expression. B. Quantification of LAMP1 puncta and total cellular LAMP1 staining intensity in U2OS upon treatment with small interfering RNA targeting INPP4B or a non-specific control. C. U2OS cells transfected with mCherry or INPP4BmCherry and labelled for lysosomes with Lucifer yellow, followed by spinning disc confocal microscopy. The inset is a magnified portion of field-of-view tracking Lucifer yellow lysosome(s) puncta and INPP4B-mCherry puncta. D. Quantification of mCherry and INPP4B-mCherry association with lysosomes. Data shown represent mean FH/FL + SEM from 3 independent experiments with 25-30 cells assessed per condition. E. U2OS cells transfected as above, followed by time lapse live imaging every $30 \mathrm{sec}$ for $20 \mathrm{~min}$. Fluorescence micrographs represent single z-plane images at 0, 5, 10, 15 and 20 min obtained by spinning disc microscopy. The inset is a magnified portion of field-of-view tracking Lucifer yellow lysosome(s) puncta and INPP4B-mCherry puncta. F. Quantification of INPP4B-mCherry association with lysosomes at time points $0,5,10,15$ and 20 min. Data shown represent mean FH/FL + SEM from 4 independent experiments with 1-2 cells per experiment. Unpaired student's t-test used with 
$* \mathrm{P}<0.05$ comparing data from all time points. Scale bar: $5 \mu \mathrm{m}$. G. Lysosome immunoprecipitation to isolate TMEM192-3xHA tagged lysosomes performed with U2OS cells stably transfected with TMEM192-3xHA (3xHA), INPP4B-FLAG (INPP4B), mock transfected or untransfected (Ctrl) cells. Immunoblot for protein markers of lysosomes (LAMP1) and endoplasmic reticulum (VAPB) in whole cell lysates (input) and purified lysosomes, or immunoprecipitates (pulldown). INPP4B was probed for association with lysosomes.

Supplementary Figure 7: INPP4B regulates lysosomal biology in leukemia cells. A. InducibleINPP4B OCI-AML2 cells were treated without or with doxycycline $(100 \mathrm{mg} / \mathrm{mL})$ and incubated with DQ-BSA-Green ${ }^{\mathrm{TM}}$ or Dextran-Red. Fluorescence was monitored by flow cytometry hourly, for up to 6 hours. Full time course displayed. B. Inpp $4 b^{+/+}$and Inpp $4 B^{-/-} M L L-A F 9$ leukemia blasts were incubated with and incubated with DQ-BSA-Green ${ }^{\mathrm{TM}}$ or Dextran-Red. Fluorescence was monitored by flow cytometry hourly, for up to 8 hours. Full time course displayed. C. Lys05 Dose response in Inpp $4 b^{+/+}$ and Inpp $4 B^{-/} M L L-A F 9$ leukemia blasts.

Supplementary Table 1. Differentially upregulated and downregulated genes associated with leukemia and differentiation for Inpp $4 b^{+/+}$and Inpp $4 b^{-/} M L L-A F 9$ leukemias,

Supplementary Table 2. Ranked list of Biological processes most significantly influenced by Inpp $4 b$ deficiency as determined by Gene Set Enrichment Analysis (GSEA) using significantly altered transcripts and identified changes in key pathways.

\section{References}


The Cancer Genome Atlas Network (2012). Comprehensive molecular portraits of human breast tumours. Nature 490, 61-70.

The Cancer Genome Atlas Research Network, Ley, T.J., Miller, C., Ding, L., Raphael, B.J., Mungall, A.J., Robertson, A.G., Hoadley, K., Triche, T.J., Laird, P.W., et al. (2013). Genomic and epigenomic landscapes of adult de novo acute myeloid leukemia. N. Engl. J. Med. 368, 2059-2074.

Amaravadi, R.K., and Winkler, J.D. (2012). Lys05: a new lysosomal autophagy inhibitor. Autophagy 8, $1383-1384$.

Bagshaw, R.D., Mahuran, D.J., and Callahan, J.W. (2005a). A proteomic analysis of lysosomal integral membrane proteins reveals the diverse composition of the organelle. Mol. Cell. Proteomics 4, 133-143.

Bagshaw, R.D., Mahuran, D.J., and Callahan, J.W. (2005b). Lysosomal membrane proteomics and biogenesis of lysosomes. Mol. Neurobiol. 32, 27-41.

Balgobind, B.V., Van den Heuvel-Eibrink, M.M., De Menezes, R.X., Reinhardt, D., Hollink, I.H.I.M., Arentsen-Peters, S.T.J.C.M., van Wering, E.R., Kaspers, G.J.L., Cloos, J., de Bont, E.S.J.M., et al. (2011). Evaluation of gene expression signatures predictive of cytogenetic and molecular subtypes of pediatric acute myeloid leukemia. Haematologica 96, 221-230.

Bartolomeo, R., Cinque, L., De Leonibus, C., Forrester, A., Salzano, A.C., Monfregola, J., De Gennaro, E., Nusco, E., Azario, I., Lanzara, C., et al. (2017). mTORC1 hyperactivation arrests bone growth in lysosomal storage disorders by suppressing autophagy. J. Clin. Invest. 127, 3717-3729.

Breuer, K., Foroushani, A.K., Laird, M.R., Chen, C., Sribnaia, A., Lo, R., Winsor, G.L., Hancock, R.E.W., Brinkman, F.S.L., and Lynn, D.J. (2013). InnateDB: systems biology of innate immunity and beyond--recent updates and continuing curation. Nucleic Acids Res. 41, D1228-33.

Cabukusta, B., and Neefjes, J. (2018). Mechanisms of lysosomal positioning and movement. Traffic 19, 761-769.

Callahan, J.W., Bagshaw, R.D., and Mahuran, D.J. (2009). The integral membrane of lysosomes: its proteins and their roles in disease. J. Proteomics 72, 23-33.

Cancer Genome Atlas Research Network, Ley, T.J., Miller, C., Ding, L., Raphael, B.J., Mungall, A.J., Robertson, A.G., Hoadley, K., Triche, T.J., Laird, P.W., et al. (2013). Genomic and epigenomic landscapes of adult de novo acute myeloid leukemia. N. Engl. J. Med. 368, 2059-2074.

Cechakova, L., Ondrej, M., Pavlik, V., Jost, P., Cizkova, D., Bezrouk, A., Pejchal, J., Amaravadi, R.K., Winkler, J.D., and Tichy, A. (2019). A potent autophagy inhibitor (lys05) enhances the impact of ionizing radiation on human lung cancer cells H1299. Int. J. Mol. Sci. 20.

Chi, M.N., Guo, S.T., Wilmott, J.S., Guo, X.Y., Yan, X.G., Wang, C.Y., Liu, X.Y., Jin, L., Tseng, H.Y., Liu, T., et al. (2015). INPP4B is upregulated and functions as an oncogenic driver through SGK3 in a subset of melanomas. Oncotarget 6, 39891-39907.

Dick, J.E. (2008). Stem cell concepts renew cancer research. Blood 112, 4793-4807.

Dzneladze, I., He, R., Woolley, J.F., Son, M.H., Sharobim, M.H., Greenberg, S.A., Gabra, M., Langlois, C., Rashid, A., Hakem, A., et al. (2015). INPP4B overexpression is associated with poor clinical 
outcome and therapy resistance in acute myeloid leukemia. Leukemia 29, 1485-1495.

Dzneladze, I., Woolley, J.F., Rossell, C., Han, Y., Rashid, A., Jain, M., Reimand, J., Minden, M.D., and Salmena, L. (2018). SubID, a non-median dichotomization tool for heterogeneous populations, reveals the pan-cancer significance of INPP4B and its regulation by EVI1 in AML. PLoS ONE 13, e0191510.

Ebner, M., Koch, P.A., and Haucke, V. (2019). Phosphoinositides in the control of lysosome function and homeostasis. Biochem. Soc. Trans. 47, 1173-1185.

Eppert, K., Takenaka, K., Lechman, E.R., Waldron, L., Nilsson, B., van Galen, P., Metzeler, K.H., Poeppl, A., Ling, V., Beyene, J., et al. (2011). Stem cell gene expression programs influence clinical outcome in human leukemia. Nat. Med. 17, 1086-1093.

Estey, E., and Döhner, H. (2006). Acute myeloid leukaemia. Lancet 368, 1894-1907.

Ferron, M., Boudiffa, M., Arsenault, M., Rached, M., Pata, M., Giroux, S., Elfassihi, L., Kisseleva, M., Majerus, P.W., Rousseau, F., et al. (2011). Inositol polyphosphate 4-phosphatase B as a regulator of bone mass in mice and humans. Cell Metab. 14, 466-477.

García-Prat, L., Kaufmann, K.B., Schneiter, F., Voisin, V., Murison, A., Chen, J., Chan-Seng-Yue, M., Gan, O.I., McLeod, J.L., Smith, S.A., et al. (2021). Dichotomous regulation of lysosomes by MYC and TFEB controls hematopoietic stem cell fate. BioRxiv.

Gasser, J.A., Inuzuka, H., Lau, A.W., Wei, W., Beroukhim, R., and Toker, A. (2014). SGK3 mediates INPP4B-dependent PI3K signaling in breast cancer. Mol. Cell 56, 595-607.

Grimwade, D., Hills, R.K., Moorman, A.V., Walker, H., Chatters, S., Goldstone, A.H., Wheatley, K., Harrison, C.J., Burnett, A.K., and National Cancer Research Institute Adult Leukaemia Working Group (2010). Refinement of cytogenetic classification in acute myeloid leukemia: determination of prognostic significance of rare recurring chromosomal abnormalities among 5876 younger adult patients treated in the United Kingdom Medical Research Council trials. Blood 116, 354-365.

Guo, S.T., Chi, M.N., Yang, R.H., Guo, X.Y., Zan, L.K., Wang, C.Y., Xi, Y.F., Jin, L., Croft, A., Tseng, H.Y., et al. (2016). INPP4B is an oncogenic regulator in human colon cancer. Oncogene 35, 3049-3061.

Herold, T., Jurinovic, V., Batcha, A.M.N., Bamopoulos, S.A., Rothenberg-Thurley, M., Ksienzyk, B., Hartmann, L., Greif, P.A., Phillippou-Massier, J., Krebs, S., et al. (2018). A 29-gene and cytogenetic score for the prediction of resistance to induction treatment in acute myeloid leukemia. Haematologica $103,456-465$.

$\mathrm{Hu}$, Y., and Smyth, G.K. (2009). ELDA: extreme limiting dilution analysis for comparing depleted and enriched populations in stem cell and other assays. J. Immunol. Methods 347, 70-78.

Inpanathan, S., and Botelho, R.J. (2019). The lysosome signaling platform: adapting with the times. Front. Cell Dev. Biol. 7, 113.

Jin, H., Yang, L., Wang, L., Yang, Z., Zhan, Q., Tao, Y., Zou, Q., Tang, Y., Xian, J., Zhang, S., et al. (2018). INPP4B promotes cell survival via SGK3 activation in NPM1-mutated leukemia. J. Exp. Clin. Cancer Res. 37, 8.

Jung, N., Dai, B., Gentles, A.J., Majeti, R., and Feinberg, A.P. (2015). An LSC epigenetic signature is 
largely mutation independent and implicates the HOXA cluster in AML pathogenesis. Nat. Commun. 6, 8489.

Klein, H.-U., Ruckert, C., Kohlmann, A., Bullinger, L., Thiede, C., Haferlach, T., and Dugas, M. (2009). Quantitative comparison of microarray experiments with published leukemia related gene expression signatures. BMC Bioinformatics 10, 422.

Klepin, H.D., Rao, A.V., and Pardee, T.S. (2014). Acute myeloid leukemia and myelodysplastic syndromes in older adults. J. Clin. Oncol. 32, 2541-2552.

Kofuji, S., Kimura, H., Nakanishi, H., Nanjo, H., Takasuga, S., Liu, H., Eguchi, S., Nakamura, R., Itoh, R., Ueno, N., et al. (2015). INPP4B is a ptdins(3,4,5)p3 phosphatase that can act as a tumor suppressor. Cancer Discov. 5, 730-739.

Korolchuk, V.I., Saiki, S., Lichtenberg, M., Siddiqi, F.H., Roberts, E.A., Imarisio, S., Jahreiss, L., Sarkar, S., Futter, M., Menzies, F.M., et al. (2011). Lysosomal positioning coordinates cellular nutrient responses. Nat. Cell Biol. 13, 453-460.

Krivtsov, A.V., Twomey, D., Feng, Z., Stubbs, M.C., Wang, Y., Faber, J., Levine, J.E., Wang, J., Hahn, W.C., Gilliland, D.G., et al. (2006). Transformation from committed progenitor to leukaemia stem cell initiated by MLL-AF9. Nature 442, 818-822.

Lara-Astiaso, D., Weiner, A., Lorenzo-Vivas, E., Zaretsky, I., Jaitin, D.A., David, E., Keren-Shaul, H., Mildner, A., Winter, D., Jung, S., et al. (2014). Immunogenetics. Chromatin state dynamics during blood formation. Science 345, 943-949.

Laurenti, E., Doulatov, S., Zandi, S., Plumb, I., Chen, J., April, C., Fan, J.-B., and Dick, J.E. (2013). The transcriptional architecture of early human hematopoiesis identifies multilevel control of lymphoid commitment. Nat. Immunol. 14, 756-763.

Laurenti, E., Frelin, C., Xie, S., Ferrari, R., Dunant, C.F., Zandi, S., Neumann, A., Plumb, I., Doulatov, S., Chen, J., et al. (2015). CDK6 levels regulate quiescence exit in human hematopoietic stem cells. Cell Stem Cell 16, 302-313.

Lawrence, R.E., and Zoncu, R. (2019). The lysosome as a cellular centre for signalling, metabolism and quality control. Nat. Cell Biol. 21, 133-142.

Lechman, E.R., Gentner, B., Ng, S.W.K., Schoof, E.M., van Galen, P., Kennedy, J.A., Nucera, S., Ciceri, F., Kaufmann, K.B., Takayama, N., et al. (2016). miR-126 Regulates Distinct Self-Renewal Outcomes in Normal and Malignant Hematopoietic Stem Cells. Cancer Cell 29, 214-228.

Leeman, D.S., Hebestreit, K., Ruetz, T., Webb, A.E., McKay, A., Pollina, E.A., Dulken, B.W., Zhao, X., Yeo, R.W., Ho, T.T., et al. (2018). Lysosome activation clears aggregates and enhances quiescent neural stem cell activation during aging. Science 359, 1277-1283.

Liang, R., Arif, T., Kalmykova, S., Kasianov, A., Lin, M., Menon, V., Qiu, J., Bernitz, J.M., Moore, K., Lin, F., et al. (2020). Restraining lysosomal activity preserves hematopoietic stem cell quiescence and potency. Cell Stem Cell 26, 359-376.e7.

Li Chew, C., Lunardi, A., Gulluni, F., Ruan, D.T., Chen, M., Salmena, L., Nishino, M., Papa, A., Ng, C., Fung, J., et al. (2015). In Vivo Role of INPP4B in Tumor and Metastasis Suppression through 
Regulation of PI3K-AKT Signaling at Endosomes. Cancer Discov. 5, 740-751.

Loeffler, D., Wehling, A., Schneiter, F., Zhang, Y., Müller-Bötticher, N., Hoppe, P.S., Hilsenbeck, O., Kokkaliaris, K.D., Endele, M., and Schroeder, T. (2019). Publisher Correction: Asymmetric lysosome inheritance predicts activation of haematopoietic stem cells. Nature 573, E5.

Löwenberg, B., Downing, J.R., and Burnett, A. (1999). Acute myeloid leukemia. N. Engl. J. Med. 341, 1051-1062.

Mandegar, M.A., Huebsch, N., Frolov, E.B., Shin, E., Truong, A., Olvera, M.P., Chan, A.H., Miyaoka, Y., Holmes, K., Spencer, C.I., et al. (2016). CRISPR Interference Efficiently Induces Specific and Reversible Gene Silencing in Human iPSCs. Cell Stem Cell 18, 541-553.

Marat, A.L., Wallroth, A., Lo, W.-T., Müller, R., Norata, G.D., Falasca, M., Schultz, C., and Haucke, V. (2017). mTORC1 activity repression by late endosomal phosphatidylinositol 3,4-bisphosphate. Science $356,968-972$.

McAfee, Q., Zhang, Z., Samanta, A., Levi, S.M., Ma, X.-H., Piao, S., Lynch, J.P., Uehara, T., Sepulveda, A.R., Davis, L.E., et al. (2012). Autophagy inhibitor Lys05 has single-agent antitumor activity and reproduces the phenotype of a genetic autophagy deficiency. Proc Natl Acad Sci USA 109, 8253-8258.

Metzeler, K.H., Hummel, M., Bloomfield, C.D., Spiekermann, K., Braess, J., Sauerland, M.-C., Heinecke, A., Radmacher, M., Marcucci, G., Whitman, S.P., et al. (2008). An 86-probe-set geneexpression signature predicts survival in cytogenetically normal acute myeloid leukemia. Blood 112, 4193-4201.

Mootha, V.K., Lindgren, C.M., Eriksson, K.-F., Subramanian, A., Sihag, S., Lehar, J., Puigserver, P., Carlsson, E., Ridderstråle, M., Laurila, E., et al. (2003). PGC-1alpha-responsive genes involved in oxidative phosphorylation are coordinately downregulated in human diabetes. Nat. Genet. 34, 267-273.

Munson, M.J., Allen, G.F., Toth, R., Campbell, D.G., Lucocq, J.M., and Ganley, I.G. (2015). mTOR activates the VPS34-UVRAG complex to regulate autolysosomal tubulation and cell survival. EMBO J. 34, 2272-2290.

Ng, S.W.K., Mitchell, A., Kennedy, J.A., Chen, W.C., McLeod, J., Ibrahimova, N., Arruda, A., Popescu, A., Gupta, V., Schimmer, A.D., et al. (2016). A 17-gene stemness score for rapid determination of risk in acute leukaemia. Nature 540, 433-437.

Notta, F., Zandi, S., Takayama, N., Dobson, S., Gan, O.I., Wilson, G., Kaufmann, K.B., McLeod, J., Laurenti, E., Dunant, C.F., et al. (2016). Distinct routes of lineage development reshape the human blood hierarchy across ontogeny. Science 351, aab2116.

Novershtern, N., Subramanian, A., Lawton, L.N., Mak, R.H., Haining, W.N., McConkey, M.E., Habib, N., Yosef, N., Chang, C.Y., Shay, T., et al. (2011). Densely interconnected transcriptional circuits control cell states in human hematopoiesis. Cell 144, 296-309.

Palmieri, M., Impey, S., Kang, H., di Ronza, A., Pelz, C., Sardiello, M., and Ballabio, A. (2011). Characterization of the CLEAR network reveals an integrated control of cellular clearance pathways. Hum. Mol. Genet. 20, 3852-3866. 
Perera, R.M., Di Malta, C., and Ballabio, A. (2019). Mit/tfe family of transcription factors, lysosomes, and cancer. Annu. Rev. Cancer Biol. 3, 203-222.

Pham, H.Q., Yoshioka, K., Mohri, H., Nakata, H., Aki, S., Ishimaru, K., Takuwa, N., and Takuwa, Y. (2018). MTMR4, a phosphoinositide-specific 3'-phosphatase, regulates TFEB activity and the endocytic and autophagic pathways. Genes Cells.

Pietras, E.M., Reynaud, D., Kang, Y.-A., Carlin, D., Calero-Nieto, F.J., Leavitt, A.D., Stuart, J.M., Göttgens, B., and Passegué, E. (2015). Functionally Distinct Subsets of Lineage-Biased Multipotent Progenitors Control Blood Production in Normal and Regenerative Conditions. Cell Stem Cell 17, 3546.

Pu, J., Guardia, C.M., Keren-Kaplan, T., and Bonifacino, J.S. (2016). Mechanisms and functions of lysosome positioning. J. Cell Sci. 129, 4329-4339.

Rapin, N., Bagger, F.O., Jendholm, J., Mora-Jensen, H., Krogh, A., Kohlmann, A., Thiede, C., Borregaard, N., Bullinger, L., Winther, O., et al. (2014). Comparing cancer vs normal gene expression profiles identifies new disease entities and common transcriptional programs in AML patients. Blood 123, 894-904.

Recher, C. (2015). INPP4B, a new player in the chemoresistance of AML. Blood 125, 2738-2739.

van Rhenen, A., Feller, N., Kelder, A., Westra, A.H., Rombouts, E., Zweegman, S., van der Pol, M.A., Waisfisz, Q., Ossenkoppele, G.J., and Schuurhuis, G.J. (2005). High stem cell frequency in acute myeloid leukemia at diagnosis predicts high minimal residual disease and poor survival. Clin. Cancer Res. 11, 6520-6527.

Rijal, S., Fleming, S., Cummings, N., Rynkiewicz, N.K., Ooms, L.M., Nguyen, N.-Y.N., Teh, T.-C., Avery, S., McManus, J.F., Papenfuss, A.T., et al. (2015). Inositol polyphosphate 4-phosphatase II (INPP4B) is associated with chemoresistance and poor outcome in AML. Blood 125, 2815-2824.

Saadatpour, A., Guo, G., Orkin, S.H., and Yuan, G.-C. (2014). Characterizing heterogeneity in leukemic cells using single-cell gene expression analysis. Genome Biol. 15, 525.

Sardiello, M., Palmieri, M., di Ronza, A., Medina, D.L., Valenza, M., Gennarino, V.A., Di Malta, C., Donaudy, F., Embrione, V., Polishchuk, R.S., et al. (2009). A gene network regulating lysosomal biogenesis and function. Science 325, 473-477.

Settembre, C., Zoncu, R., Medina, D.L., Vetrini, F., Erdin, S., Erdin, S., Huynh, T., Ferron, M., Karsenty, G., Vellard, M.C., et al. (2012). A lysosome-to-nucleus signalling mechanism senses and regulates the lysosome via mTOR and TFEB. EMBO J. 31, 1095-1108.

Shlush, L.I., Mitchell, A., Heisler, L., Abelson, S., Ng, S.W.K., Trotman-Grant, A., Medeiros, J.J.F., Rao-Bhatia, A., Jaciw-Zurakowsky, I., Marke, R., et al. (2017). Tracing the origins of relapse in acute myeloid leukaemia to stem cells. Nature 547, 104-108.

Somervaille, T.C.P., and Cleary, M.L. (2006). Identification and characterization of leukemia stem cells in murine MLL-AF9 acute myeloid leukemia. Cancer Cell 10, 257-268.

Subramanian, A., Tamayo, P., Mootha, V.K., Mukherjee, S., Ebert, B.L., Gillette, M.A., Paulovich, A., Pomeroy, S.L., Golub, T.R., Lander, E.S., et al. (2005). Gene set enrichment analysis: a knowledge- 
based approach for interpreting genome-wide expression profiles. Proc Natl Acad Sci USA 102, 1554515550.

Valk, P.J.M., Verhaak, R.G.W., Beijen, M.A., Erpelinck, C.A.J., Barjesteh van Waalwijk van DoornKhosrovani, S., Boer, J.M., Beverloo, H.B., Moorhouse, M.J., van der Spek, P.J., Löwenberg, B., et al. (2004). Prognostically useful gene-expression profiles in acute myeloid leukemia. N. Engl. J. Med. 350, $1617-1628$.

Verhaak, R.G.W., Wouters, B.J., Erpelinck, C.A.J., Abbas, S., Beverloo, H.B., Lugthart, S., Löwenberg, B., Delwel, R., and Valk, P.J.M. (2009). Prediction of molecular subtypes in acute myeloid leukemia based on gene expression profiling. Haematologica 94, 131-134.

Walter, R.B., Othus, M., Burnett, A.K., Löwenberg, B., Kantarjian, H.M., Ossenkoppele, G.J., Hills, R.K., Ravandi, F., Pabst, T., Evans, A., et al. (2015). Resistance prediction in AML: analysis of 4601 patients from MRC/NCRI, HOVON/SAKK, SWOG and MD Anderson Cancer Center. Leukemia 29, 312-320.

Wang, P., Ma, D., Wang, J., Fang, Q., Gao, R., Wu, W., Cao, L., Hu, X., Zhao, J., and Li, Y. (2016). INPP4B-mediated DNA repair pathway confers resistance to chemotherapy in acute myeloid leukemia. Tumour Biol. 37, 12513-12523.

Wouters, B.J., Löwenberg, B., Erpelinck-Verschueren, C.A.J., van Putten, W.L.J., Valk, P.J.M., and Delwel, R. (2009). Double CEBPA mutations, but not single CEBPA mutations, define a subgroup of acute myeloid leukemia with a distinctive gene expression profile that is uniquely associated with a favorable outcome. Blood 113, 3088-3091.

Zhang, F., Zhu, J., Li, J., Zhu, F., and Zhang, P. (2017). IRF2-INPP4B axis participates in the development of acute myeloid leukemia by regulating cell growth and survival. Gene 627, 9-14.

Zhang, F., Li, J., Zhu, J., Liu, L., Zhu, K., Cheng, S., Lv, R., and Zhang, P. (2019). IRF2-INPP4Bmediated autophagy suppresses apoptosis in acute myeloid leukemia cells. Biol. Res. 52, 11.

Zuber, J., Radtke, I., Pardee, T.S., Zhao, Z., Rappaport, A.R., Luo, W., McCurrach, M.E., Yang, M.-M., Dolan, M.E., Kogan, S.C., et al. (2009). Mouse models of human AML accurately predict chemotherapy response. Genes Dev. 23, 877-889. 


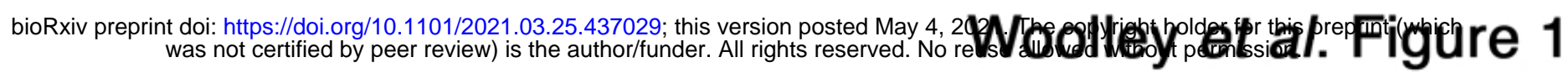
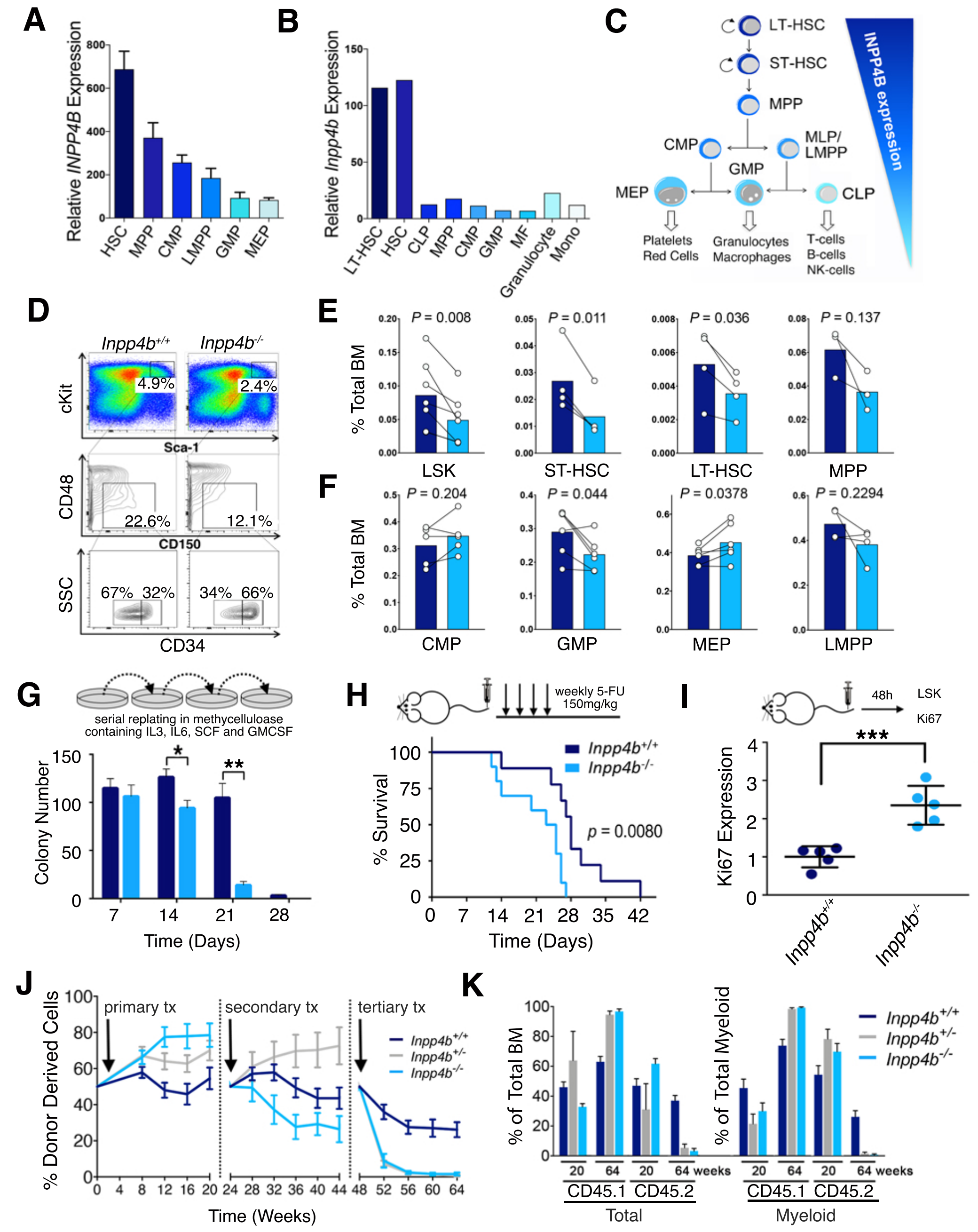


\section{Woolley et al. Figure 2}

A
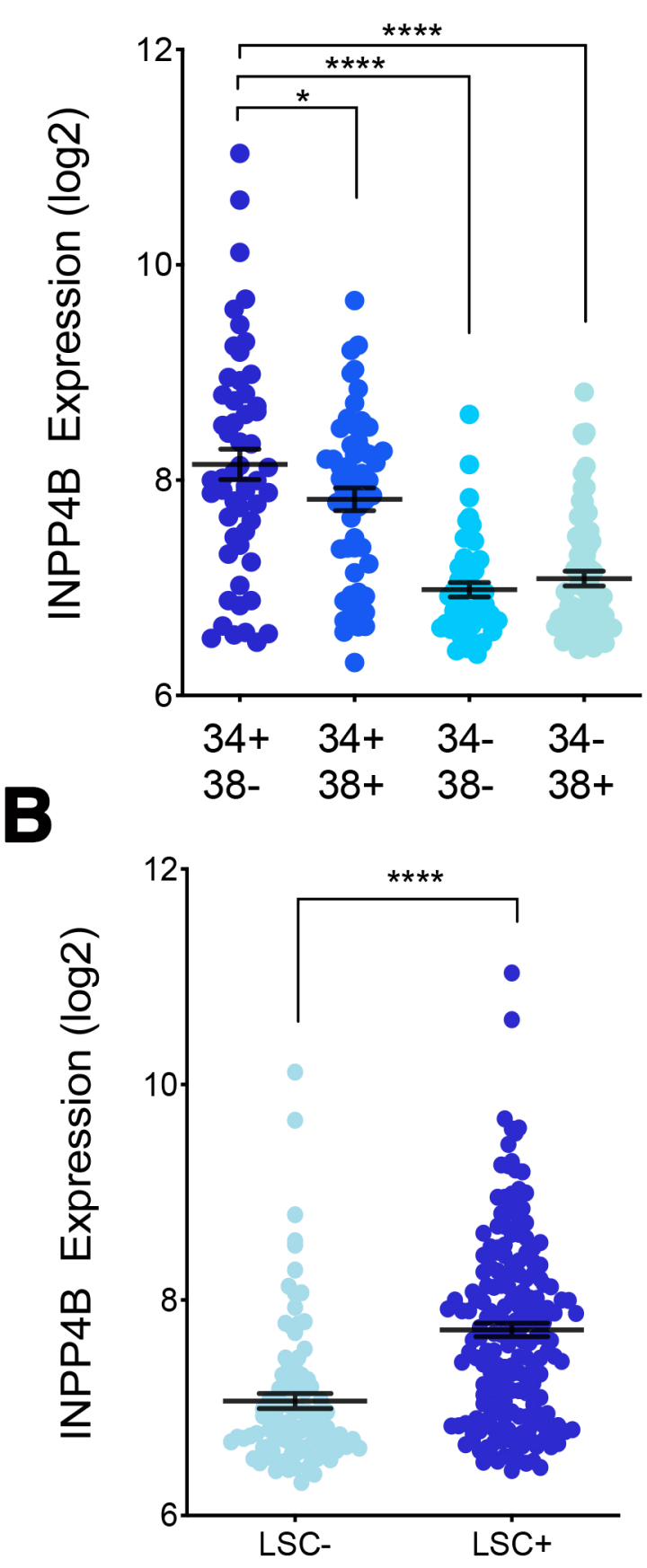

C
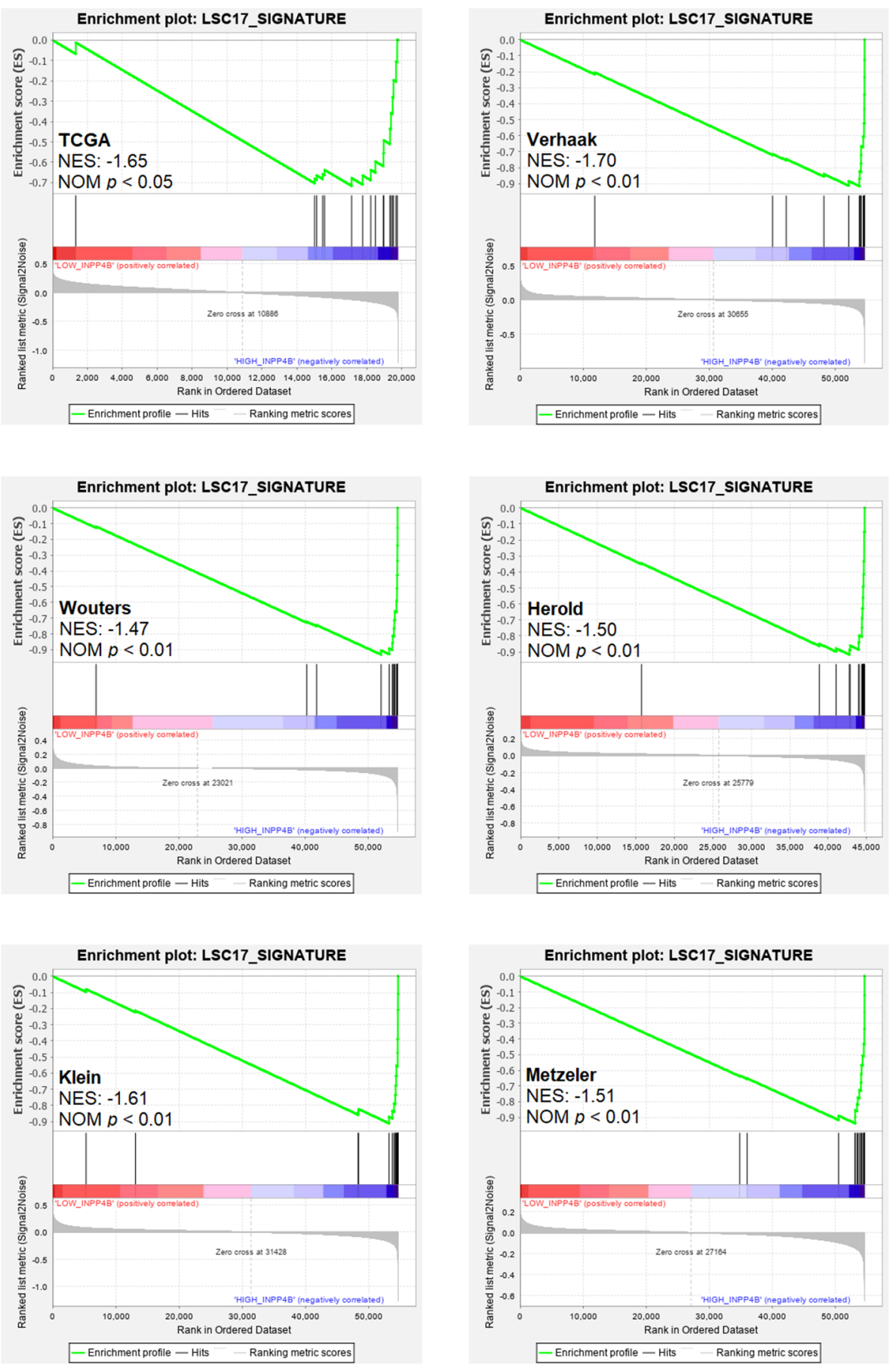
A

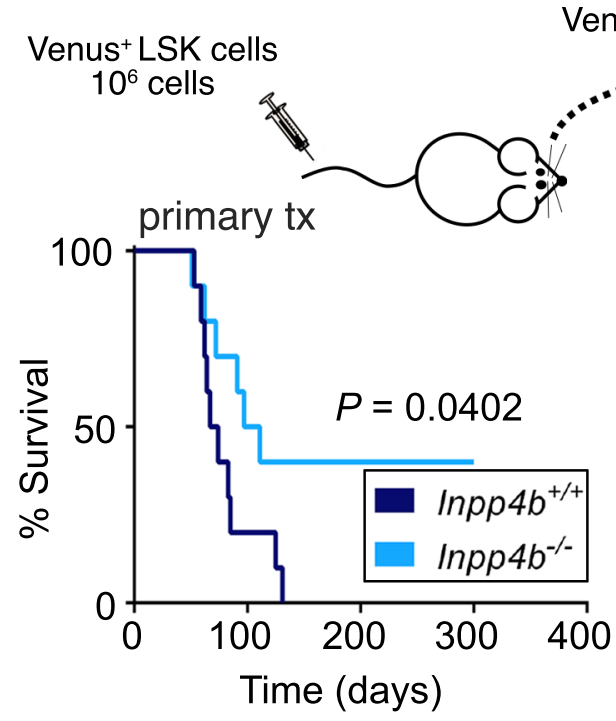

B

Venus+ MLLAF9

Leukemia
250 cells
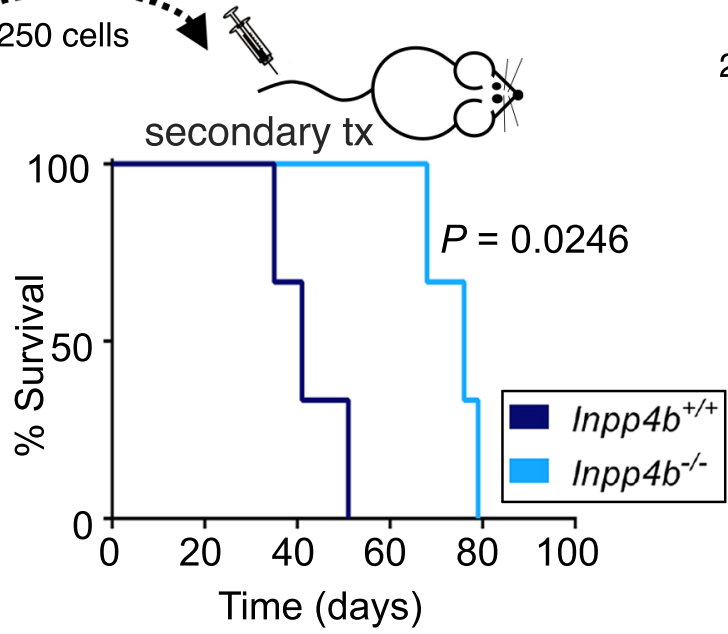

$1,10,50,100$, $250,500,1000$ cells

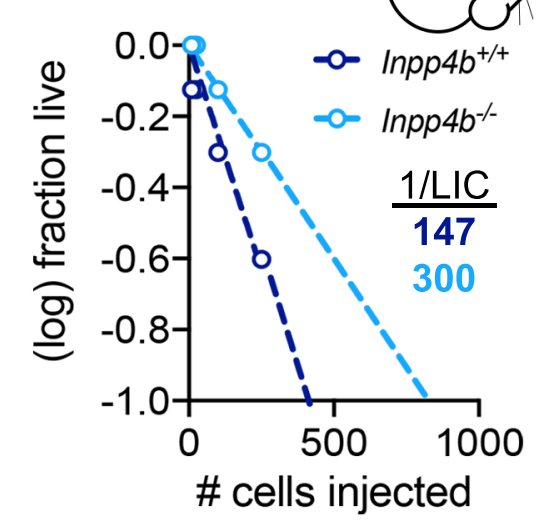

Dive Singlets:
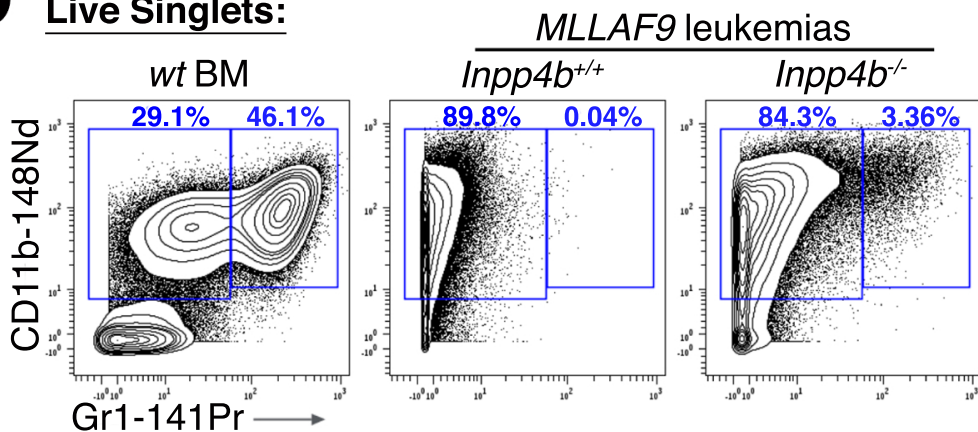

E
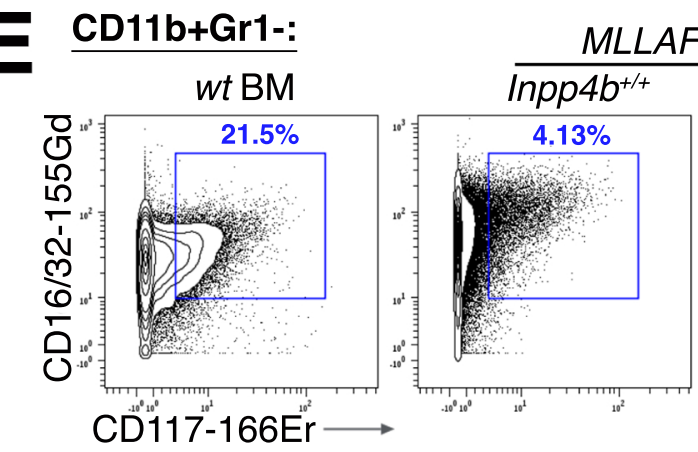

MLLAF9 leukemias

H

Colony Number

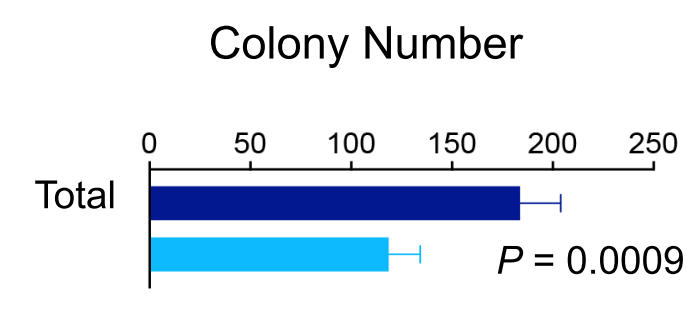

Type I
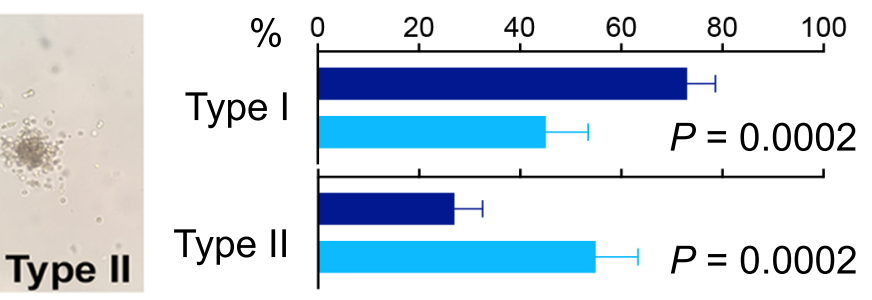
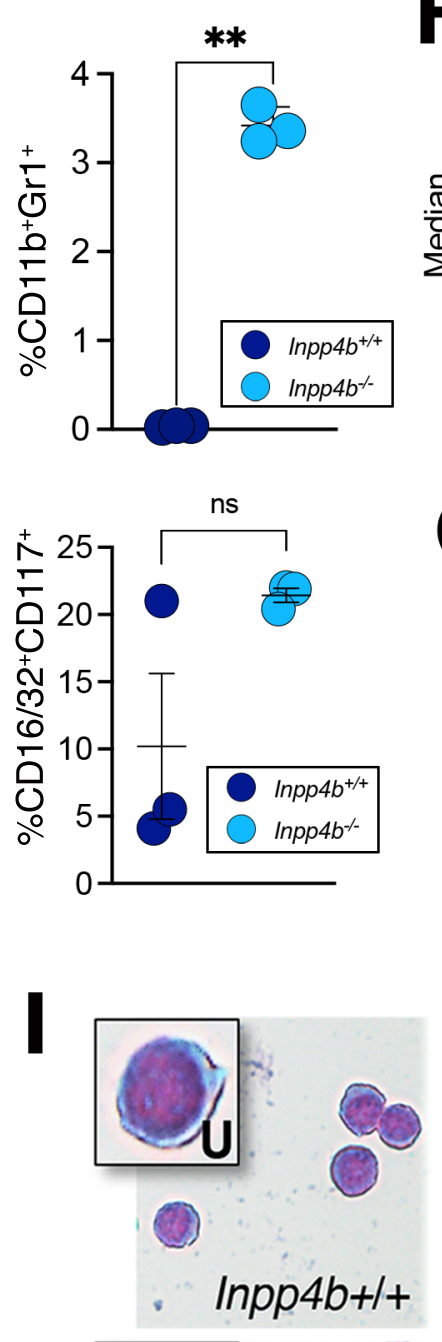

G
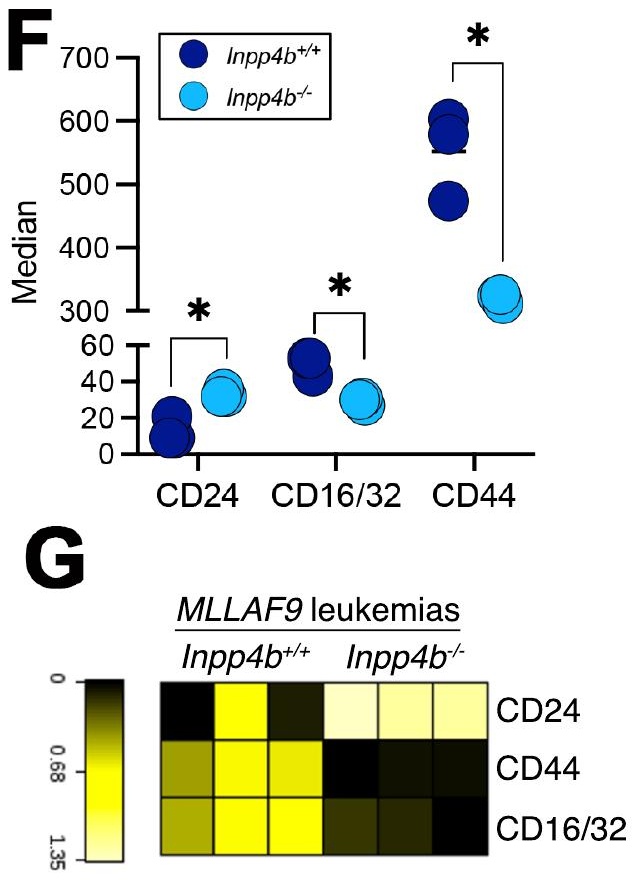

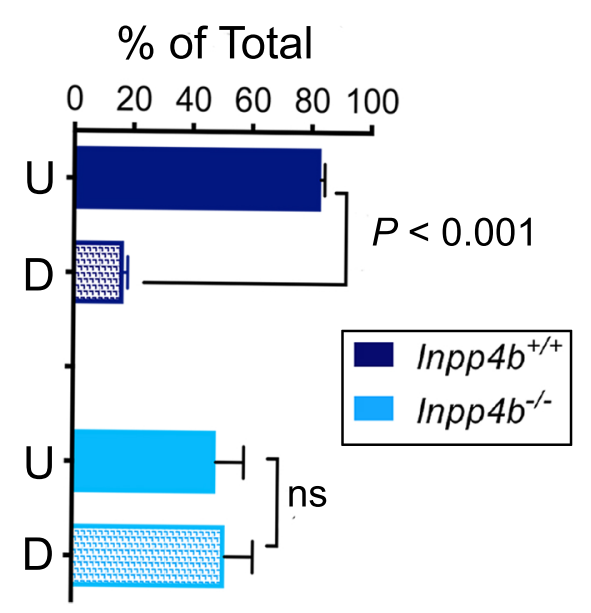


A
1-NES

\section{4 \\ 2.6}

Toll-like Receptor Signaling Lysosome Ribosome

Graft vs Host Disease Glycosaminoglycan Biosynthesis Heparan Sulfate
Allograft Rejection Arginine and Proline metabolism Cytokine Receptor Interaction Cell Adhesion Molecules B Cell Receptor Signalling Hypertrophic Cardiomyopathy Nod-lie Receptor Signaling Dilated Cardiomyopathy

Beta Alanine Metaboliem Alanine Aspartate and Glutamine Metabolism Antigen Processing and Presentation Intestinal Immune Network for IGA Production MAPK Signaling Jak-Stat Signalling Sphingolipid Metabolism -
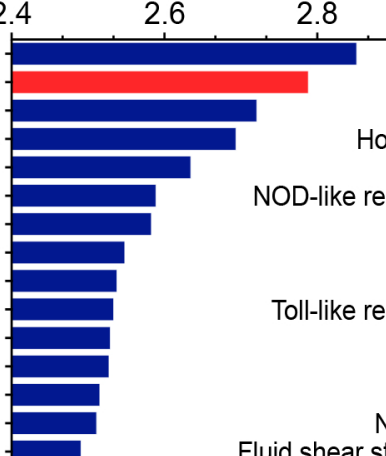

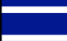

三

\section{NOD-like recep} Osteoclast differentiation ptor signaling pathway Cellular senescence

TNF signaling pathway MicroRNAs in cancer

Toll-like receptor signaling pathway Oocyte meiosis HTLV-I infection Apoptosis

Nucleotide excision repair Fluid shear stress and atherosclerosis Base excision repair Platelet activation Fanconi anemia pathway Mismatch repair

Progesterone-mediated oocyte maturation
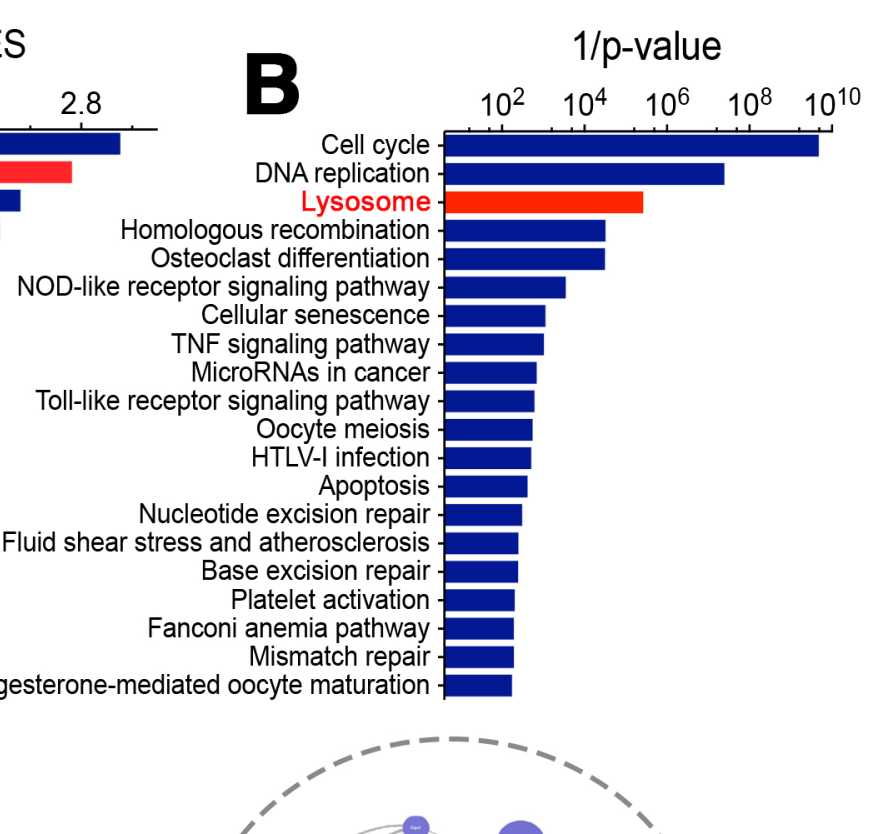

\section{$\begin{array}{lllll}-7 & -5 & -3 & -1 & 2\end{array}$}

Log Fold Change

Inverse P-value

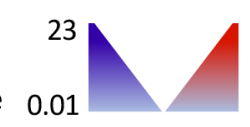

Protein-Protein Interaction

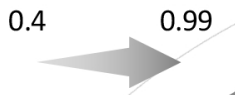

Lysosome-Active Proteins and Transporters
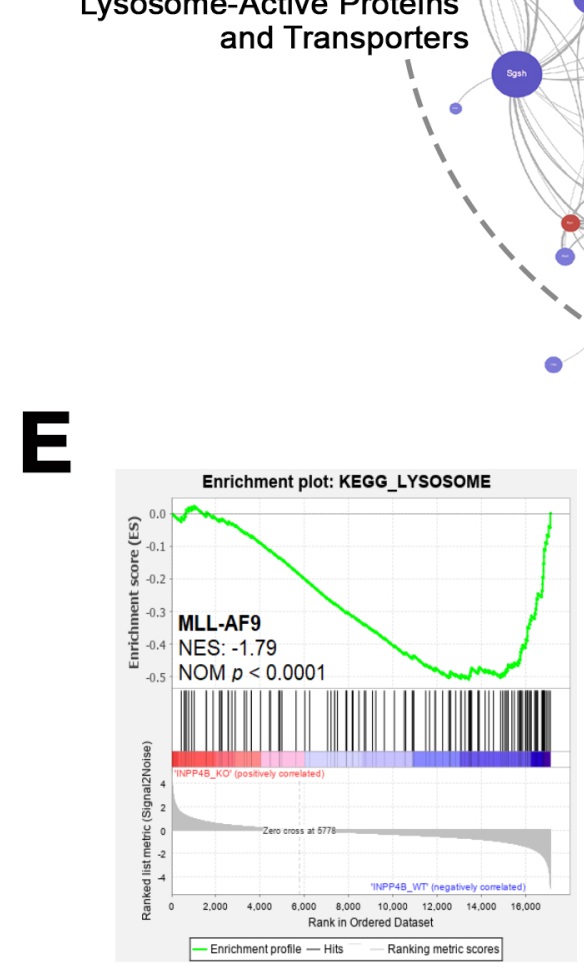
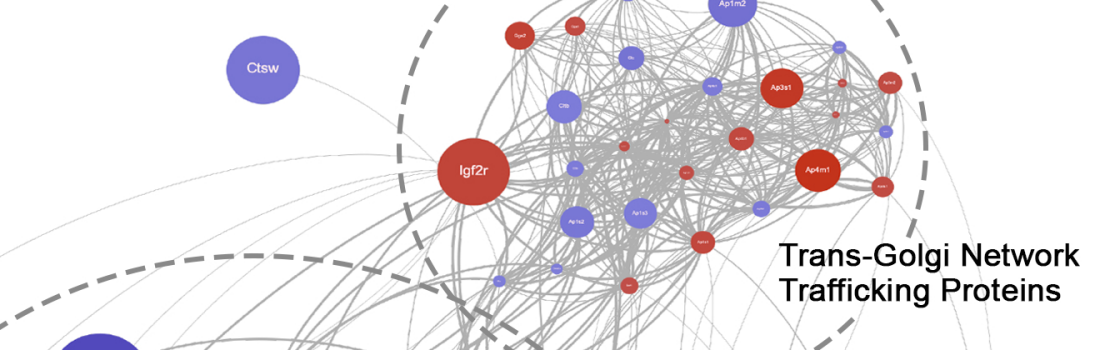


\section{Woolley et al. Figure 5}

A
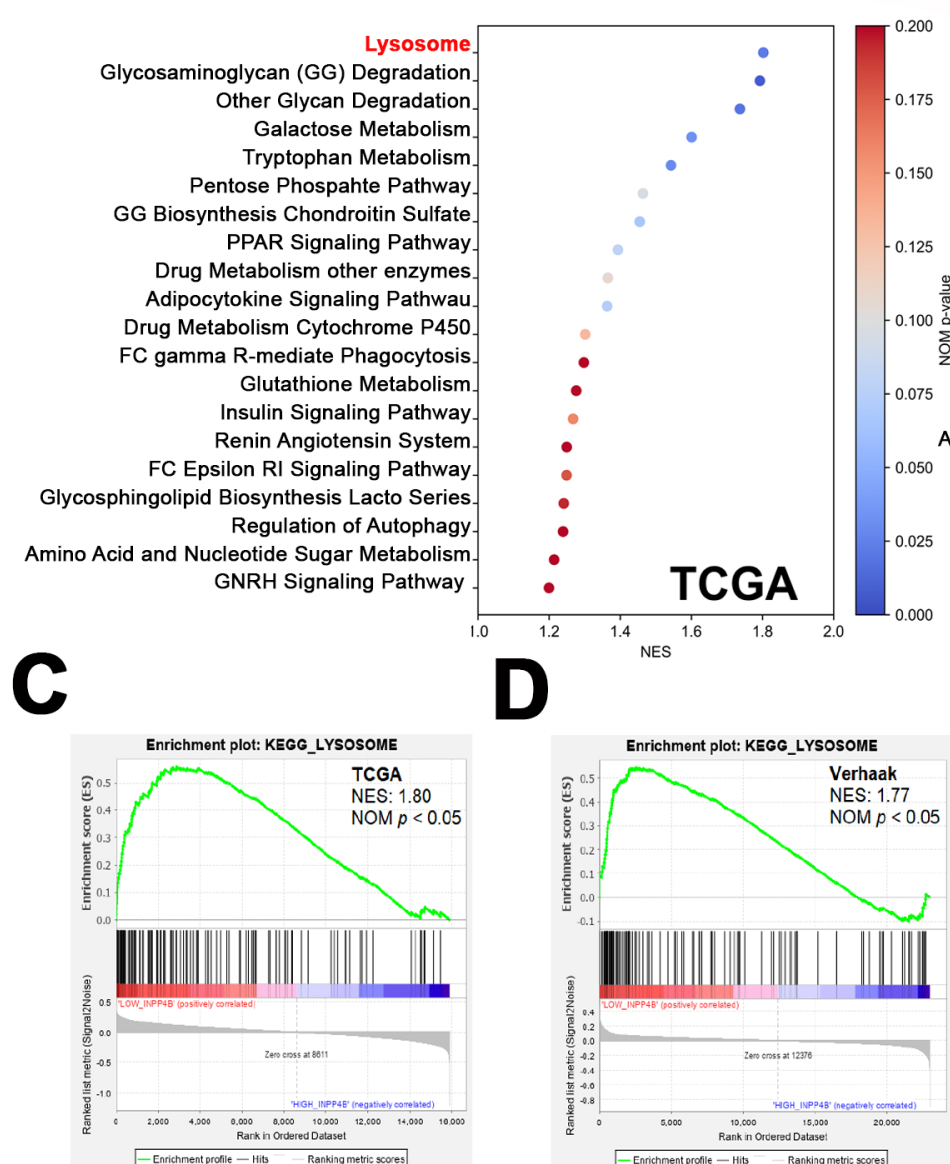

D

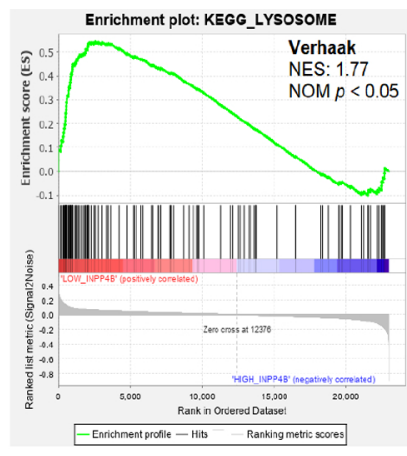

G

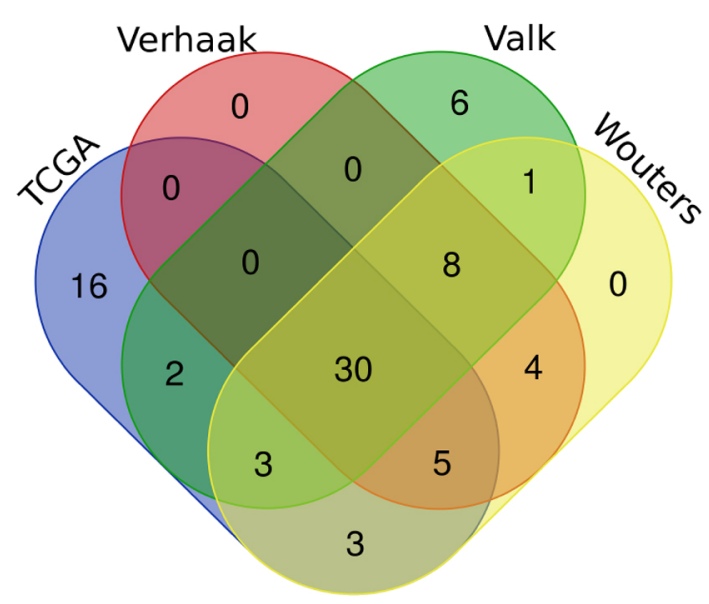

B
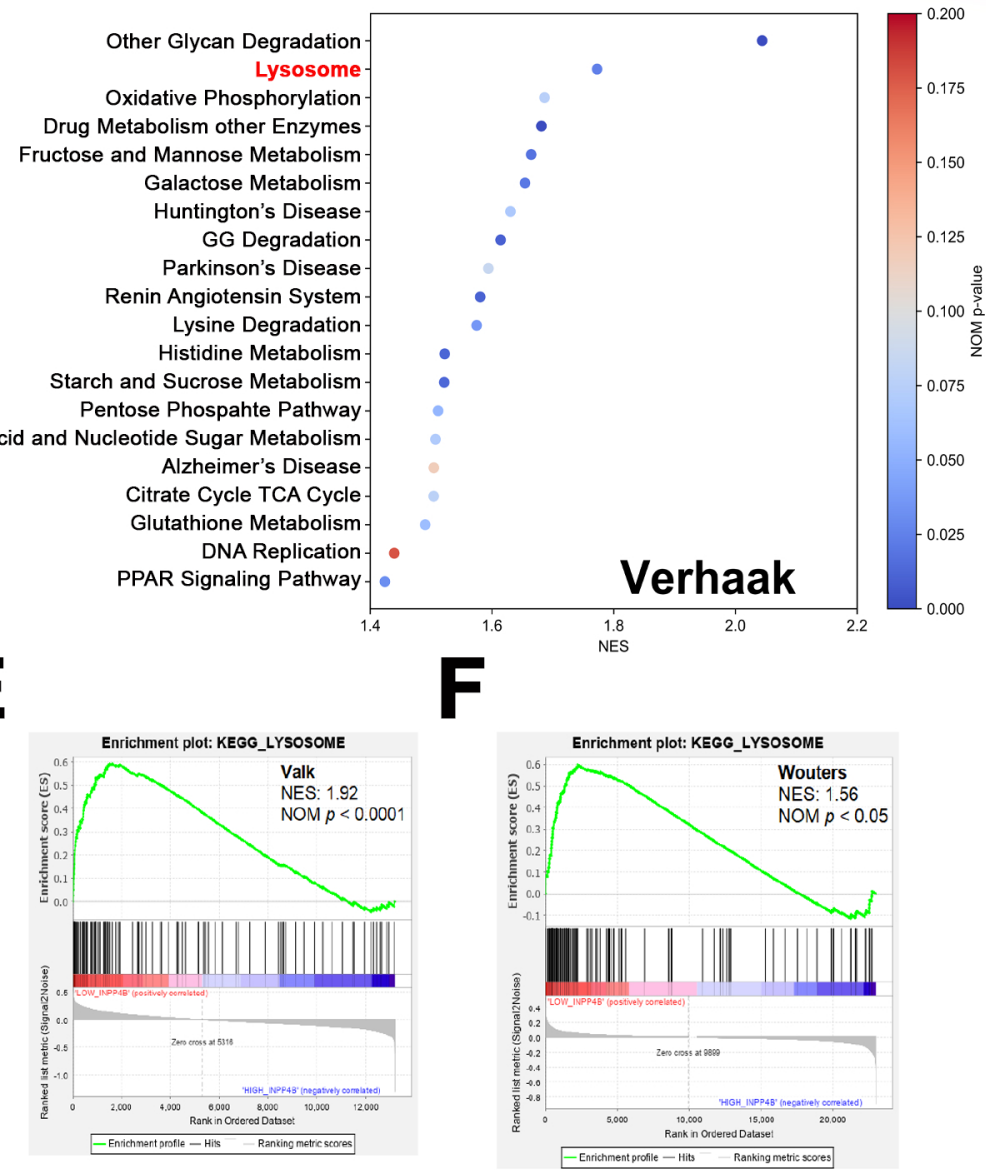

H

\begin{tabular}{|ccccc|}
\hline \multicolumn{5}{|c|}{ Core Leading Edge Lysosome Genes (30) } \\
\hline IDUA & GM2A & AGA & CTSD & HEXB \\
\hline CTSH & CTSZ & GGA2 & GALNS & NPC2 \\
\hline SLC11A1 & NAGPA & AP1S2 & CD63 & CTSA \\
\hline LAMP1 & CTSG & ATP6V0D1 & TPP1 & AP4M1 \\
\hline CLTCL1 & GLA & GNS & MANBA & GAA \\
\hline GUSB & ARSB & CTSB & ATP6V0B & PSAP \\
\hline
\end{tabular}




\section{Woolley et al. Figure 6}
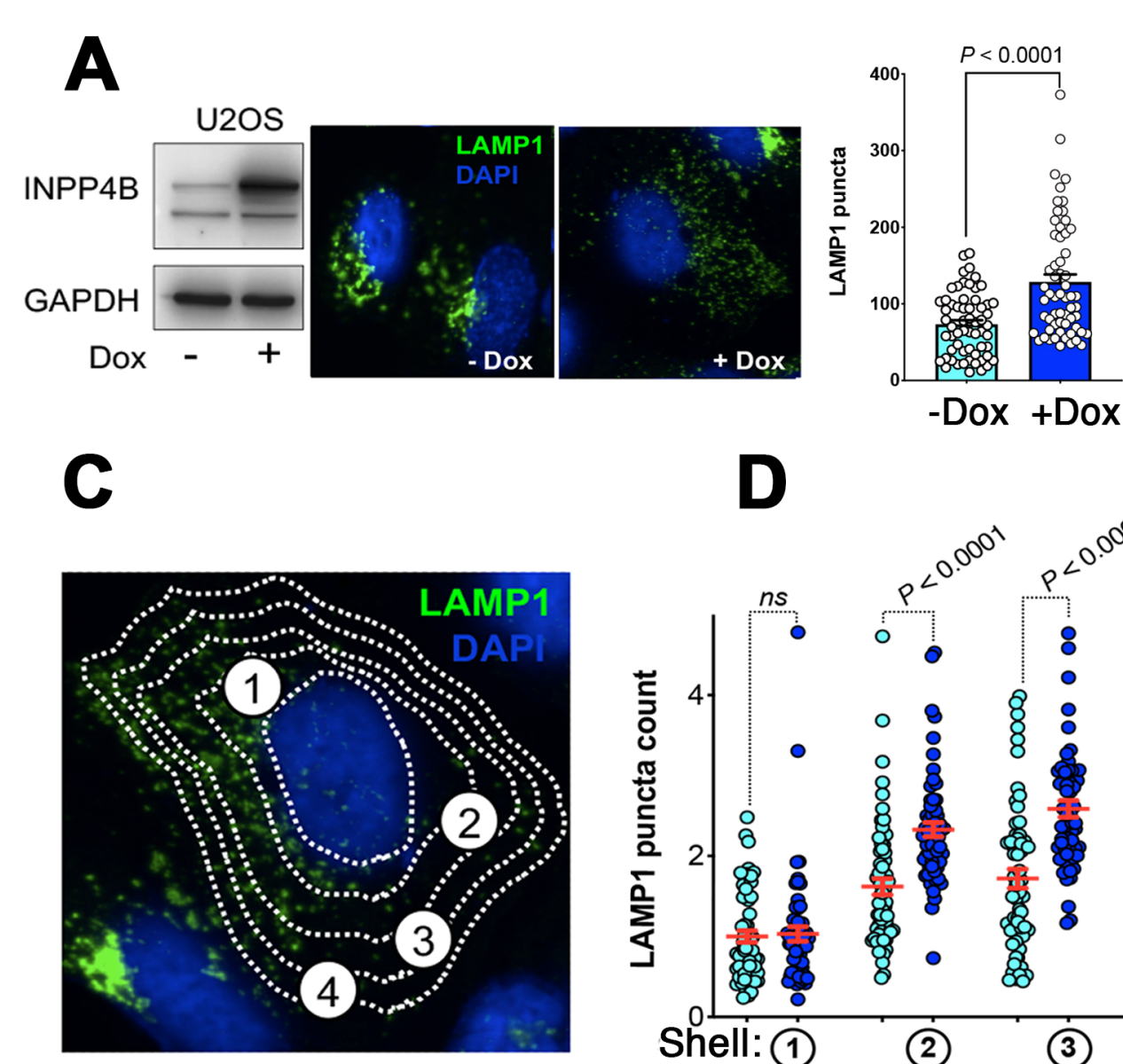

B
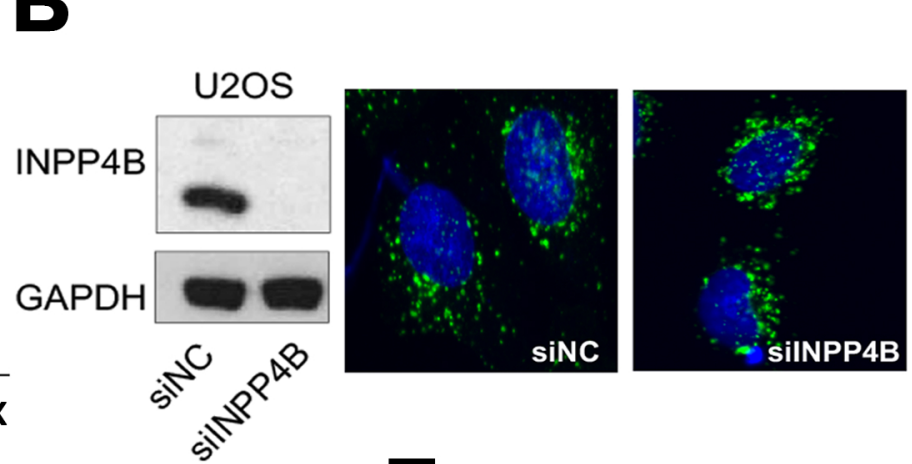

E
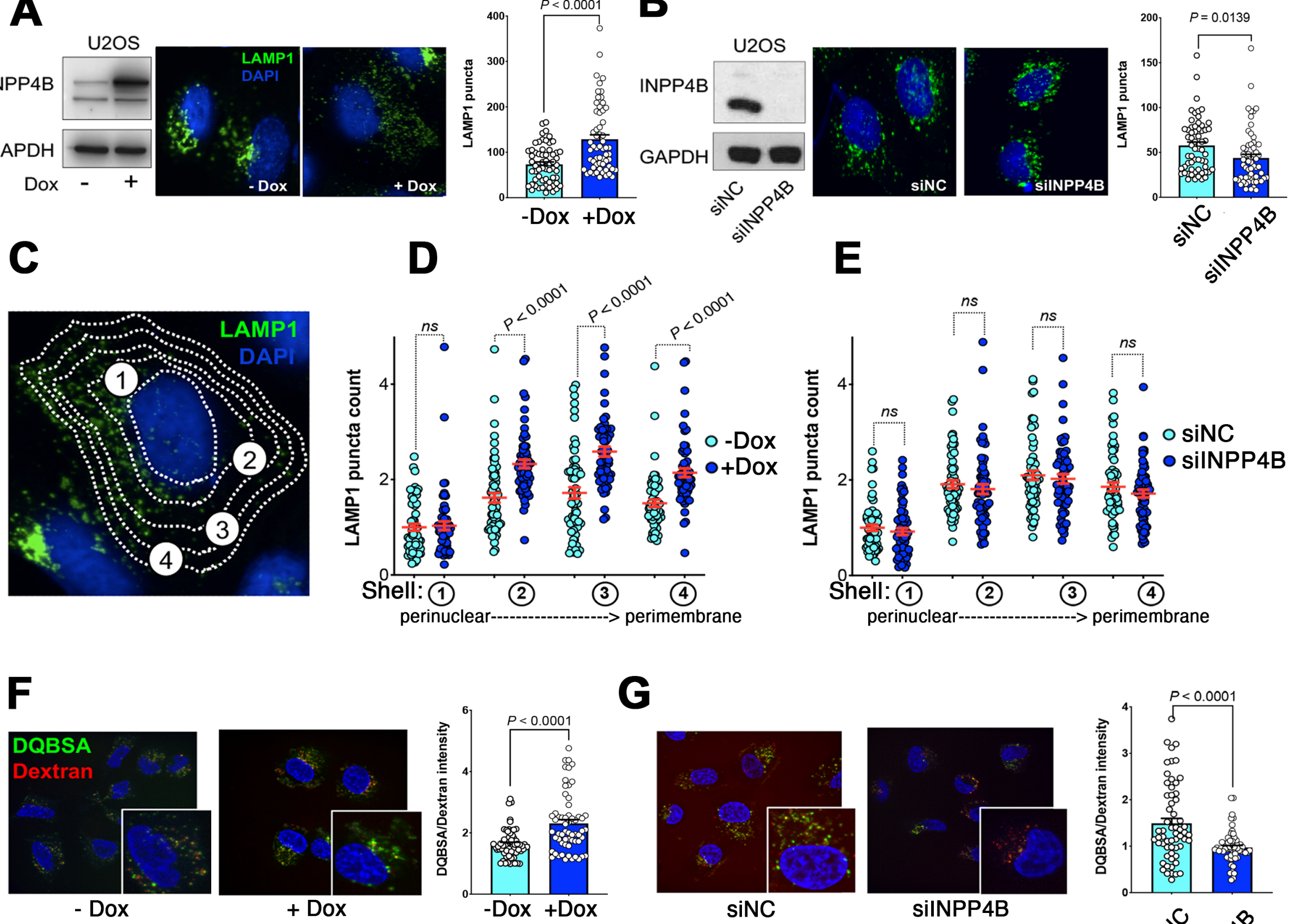

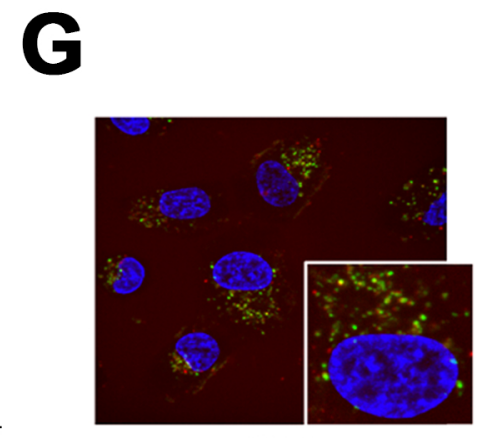

siNC

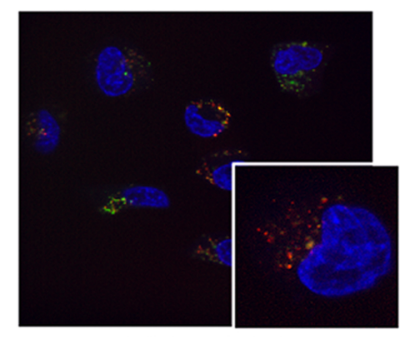

silNPP4B

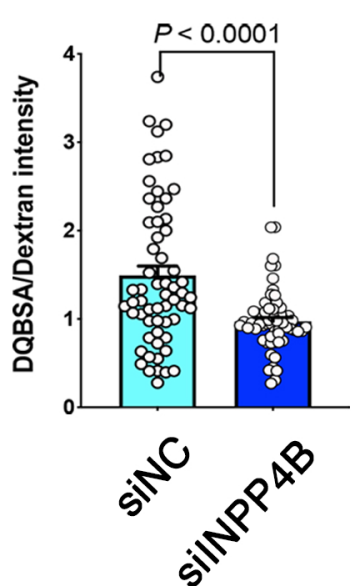


A
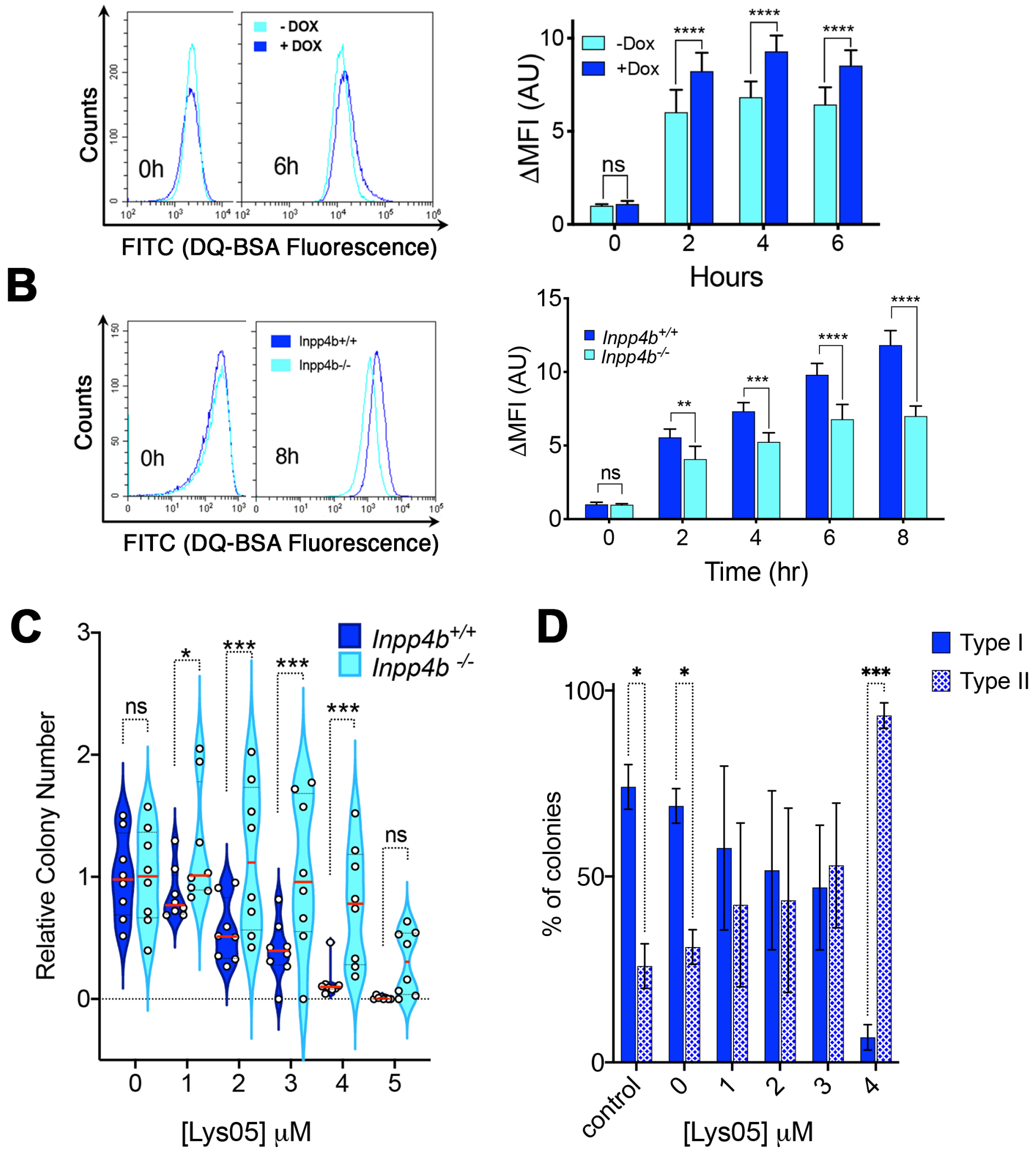